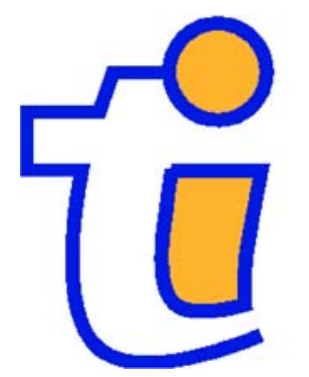

TI 2005-013/2

Tinbergen Institute Discussion Paper

Markovian Equilibrium in Infinite

Horizon Economies with Incomplete Markets and Public Policy

\author{
Manjira Datta' \\ Leonard J. Mirman² \\ Olivier F. Morand ${ }^{3}$ \\ Kevin L. Reffett ${ }^{1,4}$
}

${ }^{1}$ Arizona State University,

2 University of Virginia,

3 University of Connecticut,

4 Tinbergen Institute. 
Tinbergen Institute

The Tinbergen Institute is the institute for economic research of the Erasmus Universiteit Rotterdam, Universiteit van Amsterdam, and Vrije Universiteit Amsterdam.

Tinbergen Institute Amsterdam

Roetersstraat 31

1018 WB Amsterdam

The Netherlands

Tel.: $\quad+31(0) 205513500$

Fax: $\quad+31(0) 205513555$

Tinbergen Institute Rotterdam

Burg. Oudlaan 50

3062 PA Amsterdam

The Netherlands

Tel.: $\quad+31(0) 104088900$

Fax: $\quad+31(0) 104089031$

Please send questions and/or remarks of nonscientific nature to driessen@tinbergen.nl.

Most TI discussion papers can be downloaded at http://www.tinbergen.nl. 


\section{Markovian Equilibrium in Infinite Horizon Economies with Incomplete Markets and Public Policy ${ }^{1}$}

\author{
Manjira Datta \\ Arizona State University \\ Olivier F. Morand \\ University of Connecticut
}

\author{
Leonard J. Mirman \\ University of Virginia \\ Kevin L. Reffett \\ Arizona State University
}

January 2005

\footnotetext{
${ }^{1}$ We thank Elena Antoniadou, Alex Citanna, Jeremy Greenwood, Seppo Heikkila, Ken Judd, Tom Krebs, Michael Magill, Jianjun Miao, Martine Quinzii, Manuel Santos, Jean-Marie Viaene, Itzhak Zilcha, and especially Robert Becker, and the seminar participants at the University of Connecticut, CORE, Erasmus University, Stanford University, University of Warwick and the 2004 NBER/NSF General Equilibrium conference at UC-Davis as well as an anonymous referee and a guest editor of this journal for helpful comments and discussions. Datta thanks CORE for its gracious support of her research during her stay in spring 2002, and the Dean's Award for Excellence Summer Grant program at ASU. Reffett thanks the Tinbergen Institute at Erasmus University for its gracious support of his research during his stay in spring 2002.
} 


\begin{abstract}
We develop an isotone recursive approach to the problem of existence, computation, and characterization of nonsymmetric locally Lipschitz continuous (and, therefore, Clarke-differentible) Markovian equilibrium for a class of infinite horizon multiagent competitive equilibrium models with capital, aggregate risk, public policy, externalities, one sector production, and incomplete markets. The class of models we consider is large, and examples have been studied extensively in the applied literature in public economics, macroeconomics, and financial economics. We provide sufficient conditions that distinguish between economies with isotone Lipschitizian Markov equilibrium decision processes (MEDPs) and those that have only locally Lipschitzian (but not necessarily isotone) MEDPs. As our fixed point operators are based upon order continuous and compact nonlinear operators, we are able to provide sufficient conditions under which isotone iterative fixed point constructions converge to extremal MEDPs via successive approximation. We develop a first application of a new method for computing MEDPs in a system of Euler inequalities using isotone fixed point theory even when MEDPs are not necessarily isotone. The method is a special case of a more general mixed monotone recursive approach. We show MEDPs are unique only under very restrictive conditions. Finally, we prove monotone comparison theorems in Veinott's strong set order on the space of public policy parameters and distorted production functions.
\end{abstract}




\section{Introduction}

Over the last two decades researchers in macroeconomics and financial economics have proposed a number of new theoretical frameworks in an effort to assess the role of behavioral heterogeneity and incomplete markets on both the positive and the normative aspects of equilibrium economic fluctuations. Such models have proven to be important for researchers studying the interplay between public policy, incomplete markets, income risk (both aggregate and idiosyncratic), asset pricing, production uncertainty, quantitative welfare assessments of the costs of business cycles, financial economies, and macroeconomics. With a few notable exceptions, a majority of this work has focused on numerical characterizations of Markovian equilibrium. In addition even when theoretical work on Markovian equilibrium has been done, there has been little relationship provided to connect theoretical constructions that have used primarily pure topological fixed point methods to the study of numerical solutions for these models. This paper makes a significant contribution by bridging this large gap between theory and numerical applications for this important class of equilibrium models of economic fluctuations with behavioral heterogeneity, incomplete markets, public policy, and bounded production nonconvexities. In doing so, we suggest the possibility of broader applications of monotone methods to more complicated dynamic economies.

The problems associated with the tractability of heterogeneous agent economies (both theoretical and applied) from a mathematical perspective are well-known. One key conceptual question concerns the structure (or existence) of Markovian equilibrium in a multi-asset model. In models with many assets, little is known about the Markovian equilibrium with incomplete market where the second welfare theorem fails, production is nonconvex, and there is public policy. ${ }^{1}$ In the single-asset incomplete markets model of Bewley [13], there have been two versions of the model proposed. On the one hand, an important version of the Bewley model is presented in the work of Aiyagari [1], Krusell and Smith [52], and Miao [55]. A key feature of this class of single-asset models is that agents face a continuum of uninsured (agent specific) income risks without facing an aggregate risk. In some versions of the model, aggregate sources of risk in production are allowed, and agents have at their disposal only a single asset (e.g., capital or fiat money) for which to self insure all sources of uninsured risk. In these models many computational problems are yet to be resolved (e.g., the approximation of a probability measure and/or the existence of Markovian equilibrium decision processes and Stationary Markovian equilibrium on the so-

\footnotetext{
${ }^{1}$ One exception is Kubler and Schmedders [53] where the issue of the existence of stationary Markov equilibrium in simple multi-asset incomplete markest modle with collateral constraints is addressed. Their paper suggests the possibility of developing a general isotone iterative method based on "set-to-set" maps for computing Markov equilibrium. We remark first their methods do not work in the present single-asset context.

Reffett [63] shows that the Kubler-Schmedder's approach is a special case of a more general monotone-map method based on interval condensing methods. Numerical methods for computing such set-to-set maps in principle can be based upon the methods of Rockafellar and Wets [65].
} 
called "natural state space"). Recently, some existence issues have been resolved in a paper by Miao [55] where he proves the existence of stationary Markovian equilibrium in the model of Krusell and Smith [52] on an "expanded" state space. For this argument, the topological methods are very simple and involve a straight-forward application of a local convexity argument to the space of probability measures. ${ }^{2}$ Unfortunately from our perspective, as these topological fixed point methods are nonconstructive, it is not clear how to tie Miao's work directly to numerical approximation. In Mirman, Reffett, and Stachurski [59], some of these issues are addressed; however, those methods are not directly applicable to the economy studied in this paper. ${ }^{3}$

A second type of heterogeneous agent model having many of the features of the model of Bewley [13] has been proposed. This model is based on a stochastic version of the Ramsey model originally presented in the seminal work of Becker [4]. This class of models has the particular advantage that their state variables can be represented by a collection of functions. This is very appealing from a numerical perspectives as the solution methods can, in theory, be related to existing solution methods for systems of differential equations often encountered in the study of physics. In these models (as in the Bewley model), households face an uninsured income fluctuation problem. This problem does not have the complication of a continuum of idiosyncratic risk ex post. Therefore, in this setting, there is a finite number of households ex post and ex ante. Recursive versions of these models have been extended in a deterministic setting by Becker [5][6] (see Becker and Boyd [7] for a review of this literature) and Townsend [79], and in a stochastic setting in Scheinkman and Weiss [74], Becker and Zilcha [8], Judd and Gasper [43], Judd, Kubler, and Schmedders [44][45], and Kubler, and Schmedders [53]. In related work, a class of multisector, stochastic, incomplete markets models in the "Ramsey style" has been developed in a series of interesting papers by Krebs [49][50]. In this work, the use of a two sector incomplete markets economy with a finite number of agents that have access to both physical and human capital, but face uninsured income risk is introduced. However, Krebs studies a very specialized CES class of economies with no public

\footnotetext{
${ }^{2}$ The approach of Miao [55] is remarkably simple conceptually, and reminiscent of the approach taken for studying the existence of Markov equilibrium in large anonymous games as Bergin and Bernhardt [12]. Basically, given a compact support for the candidate sequence of probability measures, the space of probability measures on this compact support can be shown to be a weakly compact metric space. Then assuming all noise satisfies standard Feller properties, and after using a version of the LLN to aggregate of decision rules, one obtains a weakly continuous operator from the sequence of probability measures into itself. Existence then follows from a standard application of the Schauder-Tychonoff theorem (with, if necessary, one more step to construct payoff-equivalent Markovian equilibrium on an expanded state space including the "natural" state space and current period value functions). To date, this approach has had little to say about the structure of equilibrium policy functions which seems needed to obtain characterizations of numerical solutions for stationary Markov equilibrium.

${ }^{3}$ In Mirman, Reffett, and Stachurski [59], it is shown for the economies studied in Miao [55] that a new mixed-monotone map approach can compute the Markovian equilibrium decision process for such models as Aiyagari [1] and Krusell and Smith [52] using two step construction. In this paper, a much stronger existence result than in Miao [55] is proved for Markovian equilibruim decision processes on the "natural" state space as in Krusell and Smith [52].
} 
policy. In this setting, he provides a topological proof of existence of a Markovian equilibrium with no-trade.

One problem with the latter class of models is that little is known about the nature of Markovian equilibrium decision processes (or MEDPs, in short). A key innovation of this paper is to provide (i) a sharp characterization of MEDPs for each type of agent, and (ii) a description of a collection of isotone iterative methods for computing a particular Markov equilibrium from an initial guess for an MEDP (namely, an extremal MEDP). To accomplish this, we develop an application of order theoretic fixed point theory which is broadly related to the "Monotone-Map" methods for homogeneous agent economies. The complications introduced by heterogeneity are formidable. For the homogeneous agent case, the monotone map methods are found in a series of papers beginning with Bizer and Judd [15] and Coleman [19], and continuing with Greenwood and Huffman [33], Datta, Mirman and Reffett[24] and Mirman, Morand, and Reffett [58]. The work in these papers can be viewed as a collection of methodological precursors to our work for heterogeneous agent economies. ${ }^{4}$ This strand of literature, for the homogenous agent case, establishes existence and provides sufficient conditions for the global uniqueness of equilibrium within a large class of continuous Markovian equilibrium. In addition, it provides strong suggestions for computational methods for applications. Unfortunately, none of these existing methods for studying homogeneous agent economies work directly in analyzing nonsymmetric equilibrium in a multiagent setting. Our paper sets up an application of a order continuous version of the fixed point theory on complete lattices due to Tarski [76][77] to study the existence and computation of continuous Markov equilibrium.

In this paper, we provide results for heterogeneous agent economies using an integrated topological and order-theoretic approach. We provide a set of sufficient conditions under which there exists a Markovian equilibrium within a set of equicontinuous functions. We distinguish two important cases: sufficient conditions of locally Lipschitz continuous and monotone equilibrium decisions, and sufficient conditions locally Lipschitz equilibrium decision processes. Both sets of sufficient conditions seem important. In the case of monotone MEDPs, it is well-known that long-run properties are very tractable (See for example Dubins and Freedman [28] and Bhattacharya and Lee [14]. As for the latter set of sufficient conditions, the Lipschitz continuity of MEDPs plays an important role in the approximation and limiting properties of MEDPs and stationary Markov equilibrium (see Santos and Vigo [72], Santos [69], and Santos and Peralta-Alva [71]). Therefore, both sets of sufficient conditions appear important for future work on the study and characterization stationary Markov equilibrium. Further, since our methods are constructive, we provide theoretical algorithms that could prove useful for computing extremal points of the set of Markovian equi-

\footnotetext{
${ }^{4}$ For an alternative approach, using monotone operators for deterministic Ramsey equilibrium problems, see Becker and Foias [9]. Their approach looks promising, although it is yet to be developed for stochastic Ramsey problems where more than one agent in the economy can make interior savings decisions in all states. The relationship between our method and Becker and Foias' method needs to be explored in future work.
} 
librium. As the trajectories of the operator is shown to be monotone in relevant policy parameters, we conduct monotone comparative analysis in the sense of Veinott [80] (see also Milgrom and Shannon [56]) on the entire equilibrium set for equicontinuous Markovian equilibrium. Finally, we show that the computational methods employed here converges to the unique equilibrium within the set of equicontinuous equilibrium points. In this sense, this paper provides an additional level of geometric equivalence for a particular class of heterogeneous agent, incomplete market economies with capital accumulation and inelastic labor supply. Introduction of elastic labor supply and analysis of the structure of Stationary Markov equilibrium is left for the future.

The remainder of the paper is laid out as follows. In the second section of the paper, we describe a benchmark model with incomplete markets and nonclassical production. In the third section, we describe agents' decision problems in a decentralized Markov equilibrium. Section 4 focuses on the existence and computation of monotone MEDPs with a collection of isotone recursive methods. In particular, we construct the operator and prove the existence of Markovian equilibrium decision processes and provide some basic monotone comparative analysis results on the set of MEDPs using Veinott's strong set order. In the fifth section, we provide sufficient conditions under which there exists a unique Markovian equilibrium decision process within an equicontinuous class. In section 6 , we show how the isotone recursive methods can be generalized to income processes associate with general concave production functions. We show that locally Lipschitz continuous MEDPs exist and can be computed with successive approximation algorithms. It is particularly important to point out that we develop a new "mixed" monotone approach to address the existence and com-

putation questions using an expanded state space approach. The last section of the paper concludes.

\section{A Benchmark Economy with Incomplete Mar- ket and Nonclassical Production}

We first consider a benchmark economy where we study sufficient conditions for the existence of isotone MEDPs. It is well known that the studying of the long-run properties of Markovian models is greatly simplified when the Markov processes are isotone (e.g., see Dubins and Freedman [28] and Bhattacharya and Lee [14]). To do so, we consider a multiagent version of the growth under uncertainty model originally proposed by Brock and Mirman [17], modified by Becker and Zilcha [8]. Relative to Becker and Zilcha [8], we allow for more general production processes (e.g., nonclassical technologies) and public policy (e.g., fiscal policy and monetary policy). We show that the model admits isotone MEDPs. We amend the classical stochastic production setup, a generalization of the deterministic Ramsey model of Becker [4], in a number of ways. In particular, we allow for (i) Markov shocks to both aggregate technologies and individual labor income streams, (ii) equilibrium distortions and public policy, 
(iii) production nonconvexity in social returns, and, (iv) labor income risk that is uninsured. Becker and Zilcha consider the existence of a stationary Markov equilibrium, but we focus on the existence and computation of MEDPs. Our notion of uninsured idiosyncratic income risk is related to the notion in Aiyagari [1], Krusell and Smith [52], and Mirman, Reffett, and Stachurski [59], yet different since we generate a continuum of income heterogeneity in each period. Our model represents a version of the classical income fluctuation problem proposed in Schectman and Escudero [73] and developed, in continuous time, by Scheinkman and Weiss [74]. ${ }^{5}$

Time is discrete. There are a continuum of infinitely-lived household/firm agents of each type and there are finite number of types. To abstract from the complications introduced by elastic labor supply, we assume that households do not have preferences over leisure; therefore, each agent supplies its endowment of labor in fixed supply. The households face varying forms of equilibrium distortions in addition to incomplete markets for risk-sharing and this is summarized using a reduced-form production function. The equilibrium frictions can be explicitly formulated as cases of economies with: (i) public sector tax and transfer policies, (ii) market structures consistent with monopolistic competition or (iii) production nonconvexity, for example. The interested reader might want to refer to the comprehensive discussion on alternative decentralizations of our environment found in Greenwood and Huffman [33], Datta et al [24], and Morand and Reffett [62]. For production, we assume there are a large number of identical firms are endowed with an identical stochastic production technology that is (among other things) constant returns to scale in each firm's private inputs. Unlike all aforementioned work in the literature, we allow this reduced-form aggregate production technology to also possess nonconvexities in social returns. Each individual firm produces a single perishable good for sale in a competitive market using constant returns to scale production technologies, and it rents the inputs of capital and labor in competitive factor markets. As in Becker and Zilcha [8], Scheinkman and Weiss [74], Aiyagari [1], and Krusell and Smith [52], we allow households to smooth consumption by accumulating a single asset. As this asset is assumed to be productive, we refer to it as capital.

There are $J$ types of infinitely-lived household/firm agents, each of whom owns a constant fraction of capital in a firm with constant returns to scale technology. They are endowed with an initial capital holding and a unit of labor in each period. Agents of the same type are treated identically, and are identified by the subscript $j \in \mathbf{J}=\{1,2, \ldots, J\}$. Here, $\mathbf{J}$ is a finite set of agents. For convenience (and without loss of generality), we normalize the mass of agents to be the unit interval, and further assume that $\eta_{j}=\frac{1}{J}>0$, of each type $j^{6}$

\footnotetext{
${ }^{5}$ We can incorporate period-by-period uninsured idiosyncratic shocks, as for example, in Krebs [50], into our framework without changing the structure of our argument. We choose the form of idiosyncratic risk based upon making the environment as close as possible to Becker and Zilcha [8].

${ }^{6}$ Given the nonnegative vector of equilibrium capital stocks, and given our assumptions on bounded returns to the mean capital stock, we can find an upper bound for any collection of
} 
Uncertainty comes each period in the form of a vector of exogenous shocks $\theta \in \Theta \subset R_{++}^{J+1}$. The stochastic process of shocks is assumed to follow a first-order Markov process with stationary transition matrix $\chi\left(\theta, d \theta^{\prime}\right)$. To avoid technical issues associated with measurability, we assume that $\Theta$ is denumerable (e.g., either having finite or countable cardinality.) Specifically, $\theta=\left[\theta_{\text {ind }}, \theta_{a}\right]$ where $\theta_{\text {ind }}=\left[\theta_{1}, \ldots, \theta_{J}\right] \subset R_{++}^{J}$ represents the vector of type-specific idiosyncratic shocks $\left(\theta_{j}\right.$ is received by the households of type $\left.j\right)$, and $\theta_{a} \subset R_{++}$a shock to aggregate production technology. To keep things simple, we assume that $\chi\left(\theta, d \theta^{\prime}\right)$ is such that the efficiency unit shocks do not generate aggregate risk in any state, i.e., $\sum_{j=1}^{J} \eta_{j} \theta_{j}=\sum_{j=1}^{J} \frac{\theta_{j}}{J}=\widehat{\theta}=1$, in each state of the world.

For each period and state, preferences for households of type $j$ are represented by a period utility index $u_{j}(c)$, where the commodity space is assumed to be a positive interval in $R_{+}$. Letting $\theta^{t}=\left(\theta_{1}, \ldots, \theta_{t}\right)$ denote the history of the shocks until period $t$, the household's lifetime preferences are assumed to be additively separable and defined over infinite sequences, indexed by dates and histories $c=\left(c_{\theta^{t}}\right)$, and are given as,

$$
U_{j}(\boldsymbol{\theta})=E_{0}\left\{\sum_{t=0}^{\infty} \beta_{j}^{t} u_{j}\left(c_{j t}\right)\right\},
$$

where the summation in the mathematical expectation in (1) is with respect to the probability structure of future histories of the shocks $\theta^{t}$, given the transition matrix $\chi$, and $\beta_{j} \in(0,1)$. The assumptions on the period utility function $u_{j}: R_{+} \mapsto R$ for $j=1, \ldots, J$ are standard for models where interiority is an issue, and are given as,

Assumption One: The vector of period utility functions $u(c)=\left[u_{1}\left(c_{1}\right), \ldots, u_{j}\left(c_{j}\right)\right]$ satisfy the following:

(i) Each utility function $u_{j}(c)$ is at least twice continuously differentiable, strictly increasing, strictly concave;

(ii) The derivatives $u_{j}^{\prime}(c)$ satisfy the Inada conditions,

$$
\lim _{c \rightarrow 0} u_{j}^{\prime}(c)=\infty \text { and } \lim _{c \rightarrow \infty} u_{j}^{\prime}(c)=0 .
$$

Assumption One is standard in the literature. We use it to guarantee that, in equilibrium, if the mean capital stock is positive, at least one agent has positive investment. Notice that there is heterogeneity in both period utility and the discount rate. ${ }^{7}$

Each household of type $j$ is endowed with an initial stock of capital, $k_{j 0}>$ 0 , and a unit of time. The firms are assumed to use capital and labor in the production of a single perishable output good according to a constant returns to scale technology in private inputs. We summarize the distorted

weights $\eta_{j}$ for $j \in \mathbf{J}$. We use this normalization only to facilitate formulation of the upper bound for the capital stock, in equilibrium, for any agent $j \in \mathbf{J}$.

${ }^{7}$ We remark that the proofs in Becker and Zilcha [8] show that their arguments work in a setting where both period utility and discount rates differ. 
production technology by the continuous function $f\left(k, n, K_{m}, N, \theta_{a}\right)$ where $f$ : $\mathbf{K} \times[0,1] \times \mathbf{K} \times[0,1] \times \Theta_{a} \rightarrow R_{+}$and the firm's decision for capital and labor given by $(k, n) \in \mathbf{K} \times[\mathbf{0}, \mathbf{1}]$ where $\mathbf{K} \subset R_{+}$is a compact interval (to be described immediately after Assumption Two below). Also, $\Theta_{a} \subset R_{++}$is compact, $K_{m}=\sum_{j=1}^{J} \eta_{j} K_{j}=\sum_{j=1}^{J} \frac{K_{j}}{J}$ is the mean capital stock and $N$ is the average stock of labor. Let the cross-sectional distribution of initial individual capital stocks (or, initial aggregate capital stocks) by $k_{0}=\left(k_{10}, \ldots, k_{J 0}\right) \in \mathbf{K}^{J}$ (or, $\left.K_{0}=\left(K_{10}, \ldots, K_{J 0}\right) \in \mathbf{K}^{J}\right)$. In the sequel, we are more concise about where the initial capital endowments are assumed to reside. We make the following assumptions on the function $f$ :

Assumption Two: The production function $f\left(k, n, K_{m}, N, \theta_{a}\right)$ has the following properties:

(i) $f\left(k, n, K_{m}, N, \theta_{a}\right)>0$ for all $k>0, \theta_{a} \in \Theta_{a}$ whenever $k=K, n=N$ $=1$

(ii) $f\left(k, n, K_{m}, N, \theta_{a}\right)$ is continuous, strictly increasing, twice continuously differentiable, and strictly concave in $(k, n)$ for each $\left(K_{m}, N, \theta_{a}\right)$;

(iii) there exists $\widehat{k}\left(\theta_{a}\right)>0$ such that $f\left(\widehat{k}\left(\theta_{a}\right), n, K_{m}\left(\theta_{a}\right), N, \theta_{a}\right)=\widehat{k}\left(\theta_{a}\right)$ and $f\left(k, n, K_{m}, N, \theta_{a}\right)<k$ for all $k>\hat{k}\left(\theta_{a}\right)$ for all $\theta_{a} \in \Theta_{a}, k=K$ and $n=N=1$.

Here, we assume that the production function $f$ is defined net of capital depreciation. We next interpret Assumption Two in the context of our model. The types of equilibrium distortions allowed under Assumption Two are standard in the context of a homogeneous agent nonoptimal economy (e. g., Coleman [19] and Greenwood and Huffman [33]). The assumption allows for more general production technologies than Becker and Zilcha [8] (e.g., it is a reduced form settings with production nonconvexities of the sort discussed in Romer [67], monopolistic competition, and public policy. See Greenwood and Huffman [33] for a discussion).

Additionally, under Assumption Two(iii), there exists a maximum sustainable stock $\sup _{\theta_{a}} \widehat{k}\left(\theta_{a}\right)$ for this economy. We can therefore restrict the state space for capital stock to be in $[0, \bar{k}]$ where $\bar{k}=\max \left\{K_{10}, \ldots, K_{J 0}, \sup _{\theta_{a}} \widehat{k}\left(\theta_{a}\right)\right\}$. Recalling that the distribution of aggregate capital is given by $K=\left[K_{1}, \ldots, K_{J}\right] \in$ $\mathbf{K}^{J}$, as the aggregate capital stock is bounded by $\sum_{j} \eta_{j} K_{j}$, and we impose nonnegativity constraints on each component $K_{j} \geq 0$,we can use $\bar{k}$ as an upper bound for any particular agent's capital holding in equilibrium under Assumption Two.

Denote $\mathbf{K}_{+}^{J}=\mathbf{K}^{J} \backslash \mathbf{0}$, where $\mathbf{0}$ the $J$-dimensional zero and $\mathbf{K}^{J}=\Pi_{n}[0, \bar{k}]$. Assume that all initial states for the economy have $(k, K) \in \mathbf{K}_{+}^{J} \times \mathbf{K}_{+}^{J}$. Denote the aggregate state space for the entire economy by $S=[K, \theta]=\left[K_{1, \ldots,}, K_{J}, \theta\right] \in$ $\mathbf{S}=\mathbf{K}^{J} \times \Theta$. Notice that $\mathbf{S}$ is compact. Finally, since the $j$ th household enters a period with stock $k_{j}$, the state of an individual household is $s_{j}=\left[k_{j}, S\right]$. Let the distribution of initial individual capital stocks (respectively, initial aggregate capital stocks) be $k_{0}=\left(k_{10}, \ldots, k_{J 0}\right) \in \Pi_{n}[0, \bar{k}]$ (respectively, $\left.K_{0}=\left(K_{10}, \ldots, K_{J 0}\right) \in \Pi_{n}[0, \bar{k}]\right)$ with $k_{0}=K_{0}$. 


\section{Decision Problems and Definition of Equilib- rium}

Firms hire capital and labor in competitive markets: let $r(S)$ and $w(S)$ represent the rental rate on capital and the wage rate, respectively. The technologies are allowed to be non-convex (e.g., see Romer [67]). Given these prices, profit maximizing firms solve the standard optimization problem;

$$
\Delta(S)=\max _{k \geq 0, n \in[0,1]} f\left(k, n, K_{m}, N, \theta_{a}\right)-r k-w n .
$$

Following Becker and Zilcha [8], we assume that each household owns an identical share of the firm. Under constant returns to scale technologies, anticipating an equilibrium with $k=K$ and $n=N=1$, necessary and sufficient conditions are,

$$
\begin{aligned}
w(S) & =f\left(K_{m}, \theta_{a}\right)-f_{1}\left(K_{m}, \theta_{a}\right) K_{m} \\
r(S) & =f_{1}\left(K_{m}, \theta_{a}\right),
\end{aligned}
$$

where by homogeneity property in Assumption Two, $f\left(K_{m}, \theta_{a}\right)=f\left(K_{m}, 1, K_{m}, 1, \theta_{a}\right)$ is the output and $f_{1}\left(K_{m}, \theta_{a}\right)=f_{1}\left(K_{m}, 1, K_{m}, 1, \theta_{a}\right)$ is marginal product of capital both along an equilibrium path.

We allow the government to tax both capital and wage income. Assume these policy interventions have the standard separable form:

$$
\bar{r}\left(K, \theta_{a}\right)=\left[1-\pi_{k}(S)\right] r(S) \text { and } \bar{w}\left(K, \theta_{a}\right)=\left[1-\pi_{n}(S)\right] w(S)
$$

where the vector of distortion, $\pi=\left[\pi_{k}, \pi_{n}\right]$ belongs to a function space, $\Pi$, which is a space of continuous mappings $\mathbf{S} \rightarrow[0,1] \times[0,1]$. We assume the dual partial order for a function lattice is placed on $\Pi$, i.e., $\pi^{\prime} \geq \pi$ if $\pi^{\prime}(S) \leq \pi(S)$, for all $S$. In addition, we restrict attention to $\pi$ which satisfy the regularity conditions on the space of admissible distorted prices,

Assumption Three: The vector of distortions $\pi=\left[\pi_{k}, \pi_{n}\right]$ and $f$ are such that the distorted wage $\bar{w}$ and the distorted rental rate $\bar{r}$ satisfy the conditions:

(i) (a) $\bar{r}$ is decreasing in the vector $K$ and there exists a $K^{0}$ such that (b) $\beta \int \bar{r}\left(K^{0}, \theta^{\prime}\right) \chi\left(\theta, d \theta^{\prime}\right) \leq 1$ where $\beta=\max _{j \in \mathbf{J}}\left\{\beta_{j}\right\}$;

(ii) the distorted income in equilibrium for the $j$ th household, $g_{j}\left(k_{j}, K, \theta\right)=$ $\theta_{j} \bar{w}\left(K, \theta_{a}\right)+\bar{r}\left(K, \theta_{a}\right) k_{j}+J(K, \theta)$, is increasing in $(K, \theta)$, when $k_{j}=K_{j}$, and $\lim _{K \rightarrow \mathbf{0}} g(0, K, \theta)=0$.

Here, $J(K, \theta)$ is a continuous function that describes the average (and equal across all households) lump sum transfers from the government.Assumption Three (i.a) is similar to an assumption made in Becker and Zilcha [8] except that we do not require the (reduced-form) production function to be "classical" and the distorted wage rate to be concave in the mean capital-labor ratio $K_{m} .{ }^{8}$

\footnotetext{
8 By a "classical" production technology, we are referring to the standard production case considered in Brock and Mirman [17] of a production function consistent with convex aggregate production sets.
} 
Notice that under Assumption Three (i.a) $f_{1}\left(k, 1, K_{m}, 1, \theta_{a}\right)$ to be decreasing in $k$, with $k=K$. This is the same assumption as in homogeneous agent economies of Coleman [19] and Greenwood and Huffman [33]. The interpretation is that it bounds the size of production nonconvexity in equilibrium. For the methods we use in this paper, this assumption cannot be relaxed. Further, given the recent work on the nonexistence of continuous Markovian equilibrium in homogeneous agent settings (Santos [70]) and Mirman, Morand, Reffett [58], section 4), this assumption seems necessary for the existence of continuous Markovian equilibrium. We conjecture that Assumption Three (i.a) could be relaxed if one seeks a semicontinuous Markov equilibrium that is isotone in the vector $K$. (See Mirman, Morand, and Reffett [58] for one possible attack on that problem.) We leave this discussion for future work.

Assumption Three (i.b) (along with Assumption One) guarantees that in any MEDP, at least one agent holds a strictly positive amount capital. This assumption is satisfied when $f_{1}(k, 1, k, 1, \theta)$ satisfies an Inada condition as $k \rightarrow$ 0 . We remark that neither this assumption (or for that matter Assumption One) are necessary to construct our nonlinear operator used in our fixed point construction in the next section; they are needed, however, to guarantee the existence of non-trivial Markovian equilibrium; e.g., equilibrium where output is produced in all states $(k, K) \in \mathbf{K}_{+}^{J} \times \mathbf{K}_{+}^{J}$ and, at least, one agent holds capital.

Assumption Three (ii) is a joint restriction on the reduced form production function $f$, the state space $\Theta$, the transition $\chi$, and the equilibrium distortions $\Pi$ along the equilibrium trajectory of the economy, where $k=K$. This assumption is restrictive, but it is certainly not vacuous. ${ }^{9}$ This assumption is a natural multiagent generalization of assumptions embedded in the work on representative agent models of Coleman [19], Greenwood and Huffman [33], and Datta, Mirman, and Reffett [24]. Also, Assumption Three(ii) requires that as the vector of capital stocks goes to the origin (null vector) along an income trajectory of $k=K$, the vector of household incomes $g(k, K, \theta) \rightarrow \mathbf{0} .^{10}$

Under Assumptions One-Three, we study each household's dynamic program. Each household of type $j \in J$ faces the following ex ante individual feasibility constraint given by a well-defined correspondence,

$$
\Upsilon\left(s_{j}\right)=\left\{\left(c_{j}, k_{j}^{\prime}\right): c_{j}+k_{j}^{\prime}=g_{j}\left(k_{j}, K, \theta\right), c_{j}, k_{j}^{\prime} \geq 0\right\},
$$

where $g_{j}\left(k_{j}, K, \theta\right)=\theta_{j} \bar{w}\left(K, \theta_{a}\right)+k_{j} \bar{r}\left(K, \theta_{a}\right)+J(K, \theta)$. Notice that under Assumptions Two and Three, $\Upsilon\left(s_{j}\right)$ is well-defined and expanding in $\left(k_{j}, K\right)$ in the set inclusion partial order on the powersets of $R_{+}^{2}$ (i.e., $\Upsilon\left(k_{j}, K, \theta\right) \subset \Upsilon\left(k_{j}^{\prime}, K^{\prime}, \theta\right)$

\footnotetext{
${ }^{9}$ The assumption basically says that the complimentarity (or cross elasticity) between capital and labor must majorize pointwise the concavity of the production processes in capital. Not all common production functions satisfy this assumption. Some CES productions work, but the Cobb-Douglas example studied in Becker and Zilcha [8] does not work even when there are no idiosyncratic risk (e.g., the example in their section 3). We analyze these cases and other generalizations in Section 6.

${ }^{10}$ This assumption is always satisfied if $\bar{r}$ does not satisfy an Inada condition. If $f$ and $\pi$ are such that $\bar{r}$ satisfies an Inada condition, the condition is satisfied, for example, if the distortion vector $\pi \in \Pi$ depends only on the mean aggregate capital $K_{m}$ (stated in efficiency units per capita) and the production function is Cobb-Douglas.
} 
for $\left.\left(k_{j}, K\right) \leq\left(k_{j}^{\prime}, K^{\prime}\right)\right)$. Further, since $g_{j}$ is continuous in each of its arguments, $\Upsilon\left(s_{j}\right)$ is a non-empty, compact and convex-valued, continuous correspondence.

Next, we develop each household's dynamic program. Denote by $\mathbf{C}^{+}(\mathbf{S})$ the space of bounded, positive continuous functions $h=\left(h_{1}, \ldots, h_{J}\right), h_{j}: \mathbf{K}^{J} \times \Theta \rightarrow$ $\mathbf{K}^{J}$ defined on the compact set $\mathbf{S}=\mathbf{K}^{J} \times \Theta$, and with range, $R_{+}^{J}$. We equip $\mathbf{C}^{+}(\mathbf{S})$ with the standard $C^{0}$ uniform topology and the standard component wise partial order. By a standard argument, $\mathbf{C}^{+}(\mathbf{S})$ is a complete metric space, a normal and minihedral cone of continuous functions, and finally a Banach lattice (under the sup metric topology and when endowed with the pointwise partial order induced by the cone). Define a subset of individually feasible decisions $\dot{\mathbf{H}}(\mathbf{S}) \subset \mathbf{C}^{+}(\mathbf{S})$ to be $\dot{\mathbf{H}}(\mathbf{S})=\left\{h \mid h \in \mathbf{C}^{+}(\mathbf{S}), 0 \leq h_{j} \leq g_{j}\right\}$. Then when developing dynamic programs for agents, we assume agents believe the law of motion for the distribution of aggregate capital stocks by $K=\left[K_{1}, \ldots, K_{J}\right]$ is given by the following recursive representation:

$$
K^{\prime}=h(S) ; h \in \mathbf{\mathbf { H }}(\mathbf{S}) .
$$

We can now state the decision problem for each household of type $j$ under Assumptions One-Three. Let $\mathbf{G}(\mathbf{K} \times \mathbf{S})$ be the space of continuous bounded increasing functions $v(k, S): \mathbf{K} \times \mathbf{S} \rightarrow \mathbf{R}$ that are concave in $k$. Given the state variable $s_{j}$ at the beginning of any period, the decision problem for the individual of type $j$ is represented as the solution of the dynamic programming problem summarized by the Bellman equation for any $h \in \mathbf{H}(\mathbf{S})$,

$$
v_{j}\left(s_{j}\right)=\sup _{\left(c_{j}, k_{j}^{\prime}\right) \in \Upsilon\left(s_{j}\right)}\left\{u_{j}\left(c_{j}\right)+\beta_{j} \int_{\Theta} v_{j}\left(s_{j}^{\prime}\right) \chi\left(\theta, d \theta^{\prime}\right)\right\} .
$$

Appealing to the contraction mapping theorem, the existence of a function $v_{j} \in$ G that satisfies the functional equation (4) can be established; also, the function $v_{j}(s ; h)$ is increasing in $\left(k_{j}, K\right)$ and strictly concave in its first argument (as we have unbounded returns, see Duran [31]). From Mirman and Zilcha [57], the strict concavity of $v_{j}$ in its first argument implies that an envelope theorem is available, and $v_{j}$ is at least once differentiable in $k_{j}$ for each $j$.

We now define equilibrium.

Definition 1 A Markovian (or recursive) competitive equilibrium decision process $(M E D P)$ consists of initial states $\left(k_{0}, K_{0}\right) \in \mathbf{K}^{J} \times \mathbf{K}^{J}$ such that $k_{0}=K_{0} ; a$ collection of function $h^{*}(S)$ and value functions $v_{j}\left(K_{j}, S\right)$ with associated individual decisions $c_{j}^{*}\left(K_{j}, S\right)=y_{j}\left(K_{j}, S\right)-h_{j}^{*}(S)$ and $k_{j}^{* \prime}\left(K_{j}, S\right)=h_{j}^{*}(S)$ are such that: (i) given $h^{*}, v_{j}\left(K_{j}, S\right)$ is the value function for each household type $j$ that satisfies the household's Bellman equation for each type of agent $j=\{1,2, \ldots, J\}$; (ii) for each $(K, \theta)$, given the input prices, $w$ and $r$ in equations (2) and (3), the firms solve $\max _{k \geq 0, n \in[0,1]} f\left(k, n, K_{m}, N, \theta_{a}\right)-r k-w n$; (iii) all markets clear, that is, by Walras' law $k^{* \prime}(s)=h^{*}(S)=K^{\prime} \in \mathbf{H}, c^{*}(s)=C(S)=g-h^{*}$ with $\left.c_{j}^{*}\left(K_{j}, S\right)\right)+k_{j}^{* \prime}\left(K_{j}, S\right)=g_{j}\left(K_{j}, K, \theta\right)$ for all $j$; and, (iv) the government budget is balanced, that is, $J(S)=\pi_{n}(S) w(S)+\pi_{k}(S) r(S)$. 


\section{Computing Isotone MEDPs}

Prior to considering the existence of an MEDP for economies satisfying Assumptions One-Three, we define some terms that are used in subsequent discussion.

Ordered Sets and Lattices. Consider a set $X$ ordered with a reflexive, transitive, antisymmetric relation denoted by " $\geq$ ". $X$ is a partially ordered set (or Poset). An upper (lower) bound for a set $B \subset X$ is an element $x^{u}\left(x^{l}\right) \in B$, such that, $\forall x \in B, x \leq x^{u}\left(x^{l} \leq x\right)$. A set $X$ is a lattice if for any two elements $x$ and $x^{\prime}$ in $X$, the pair has a least upper bound and a greatest lower bound (denoted, respectively, by $x \vee x^{\prime}$ and $x \wedge x^{\prime}$ ). A subset of a lattice, say $B \subset X$ , is a sublattice of $X$ if it contains the sup and the inf (with respect to $X$ ) of any pair of points in $B$.A lattice is complete if for any subset $B \subset X$, the least upper bound $(\sup (X))$ and a greatest lower bound $(\inf (X))$ exist in $X$. A chain $C \subset X$ is a totally ordered subset of $X$ (i.e., all pairs of elements are ordered).

Vector Lattices and Banach Lattices. A partially ordered vector space (or semi-ordered linear space) $X$ is a real vector space equipped with a partial order $\geq$ that is compatible with the following algebraic structure: (i) if $x \geq x^{\prime}$, then $x+z \geq x^{\prime}+z$, for all $z \in X$; (ii) if $x \geq x^{\prime}$, then $\alpha x \geq \alpha x^{\prime}$ for all $\alpha \geq 0$.Any partially ordered vector space that is also a lattice is referred to as a vector lattice. If the space $X$ has a norm $\|x\|_{X}$ such that $|x| \geq\left|x^{\prime}\right|$ in $X$, implies $\|x\| \geq\left\|x^{\prime}\right\|$, then we say $X$ has a lattice norm. A complete normed vector space is a Banach space. A normed vector lattice is a vector lattice equipped with a lattice norm. A normed vector lattice $X$ that is complete in the Cauchy sense, and is endowed with a lattice norm is referred to as a Banach lattice. In much of our work we use compact subsets of Banach lattices.

Cones and Order Intervals in Banach Spaces: Let $X$ be a topological space. The set $X^{+}=\{x \in X, x \geq 0\}$ is the cone of $X$. A cone $X^{+}$is solid if the interior of $X^{+}$is nonempty. In a Banach space $X$, the set $X^{+}=\{x \in X$, $x \geq 0\}$ is the order cone of $X$. In particular, $X^{+}$is a nonempty, convex, closed set that has the following two properties: (i) $x \in X^{+} \Longrightarrow \alpha x \in X^{+}$for $\alpha \geq 0$; (ii) if $x$ and $-x$ in $X^{+}, x=0$, where 0 denote the zero of the cone. The partial order induced by the cone structure of $X^{+}$has $x_{1} \geq x_{2}$ if $x_{1}-x_{2} \in X^{+}$. A cone is normal if there exists a constant $m$ such that for any $x_{1}, x_{2} \in X^{+}, \|$ $x_{1}+x_{2}\|\geq m,\| x_{i} \|=1$ for $i=1,2$. Intuitively, a normal cone can be interpreted geometrically as bounding the angle between any two unit vectors away from the number $\pi$. Therefore, a normal cone cannot become "too large". A cone is regular if every increasing and bounded order sequence in $X^{+}$has a limit in $X^{+}$. A fully regular cone has every increasing and norm bounded sequence in $X^{+}$having a limit in $X^{+}$. A fully regular cone is also regular. A regular cone is also normal. (Guo and Lakshmikantham [35], Theorem 1.2.1).

Let $[a)=\{x \mid x \in X, x \geq a\}$ be the upperset of $a$, and $(b]=\{x \mid x \in X, x \leq b\}$ the lowerset of $b$. We say $X$ is an ordered topological space if $[a)$ and (b] are closed in the topology on $X$. An order interval is defined to be $[a, b]=[a) \cap(b]$, $a \leq b$. The spaces we study are compact subsets of partially ordered topological spaces. Therefore, if $[a, b] \subset X$, and $X$ is an ordered topological space, all order 
intervals are closed. In this paper, we study fixed point problems that are set on compact order intervals in normal cones of continuous functions defined on compact sets equipped with the standard uniform topology. Therefore we need additional structure so that sequences that converge in order and/or norm, converge in the set. We compensate for the loss of regularity (of the cone) with the compactness of the order interval.

Mappings on a Lattice: Let $\left(X, \geq_{X}\right)$ and $\left(Y, \geq_{Y}\right)$ be partially ordered sets. A mapping is a relational statement between two spaces, say $X$ and $Y$. By referring to the relation as a "mapping", we consider both "point-to-point" or "point-to-set" relations. In the case of a "point-to-point" mapping, we refer to the mapping as a function or an operator. A function or operator $m: X \rightarrow Y$ will be defined to be isotone on $X$ if $m\left(x^{\prime}\right) \geq_{Y} m(x)$, when $x^{\prime} \geq_{X} x$, for $x, x^{\prime} \in X$. If $m\left(x^{\prime}\right)>_{Y}($ respectively, $<) m(x)$ when $x^{\prime}>_{X} x$ for $x, x^{\prime} \in X$, then we say $m$ is increasing (respectively, decreasing). If $m\left(x^{\prime}\right)>_{Y}$ (respectively, $<$ ) $m(x)$ when $x^{\prime} \geq_{X} x, x^{\prime} \neq x$, then we say $m$ is strictly increasing (respectively, strictly decreasing). We say $m(x)$ is antitone if $m(x) \geq_{Y} m\left(x^{\prime}\right)$ if $x^{\prime} \geq_{x} x$. If a function is either isotone or antitone, we say it is monotone. An operator is said to be pseudo-concave on $X$ if for $t \in(0,1), x \in X, x>0, A t x>t A x$. If $A$ is pseudo-concave, and $X$ is a solid cone, we then say $A$ is strongly sublinear. (See Guo and Lakshmikantham [35], Definition 2.2.2).

Let $X^{+}$be a cone in a real Banach space, and consider a transformation or an operator $A: X^{+} \rightarrow X^{+}$. We say an operator $A$ is $e$-concave if there exists non-zero $e \in X^{+}$, such that (i) for an arbitrary non-zero $x \in X^{+}$the inequalities $\alpha e \leq A x \leq \beta e$, where $\alpha$ and $\beta$ are positive, are valid and (ii) for every $x \in X^{+}$ such that $\alpha_{1}(x) e \leq x \leq \beta_{1}(x) e$ with $\left(\alpha_{1}(x), \beta_{1}(x)\right) \gg 0$, and there is a number $\eta(x, t)>0$ such that $A t x \geq(1+\eta) t A x$ for any $t \in(0,1)$. An operator is said to be pseudo-concave on $X^{+}$if for all $t \in(0,1), x \in X^{+}, x>0$, Atx $>>t A x$. Let $X^{+}$be a solid cone, the operator $A: X \rightarrow X$ is strongly sublinear if Atx $>>t A x$ for all non-zero $x \in X^{+}$and $0<t<1$. (See Guo and Lakshmikantham [35], Definition 2.2.2).

We can generalize the notion of a monotone function (or an operator) to a correspondence (or multifunction). A correspondence or multifunction is a nonempty-valued mapping, $M: X \rightarrow 2^{Y}$, e.g., a nonempty-valued "point-toset" mapping. We say a correspondence or multifunction is ascending in the set relation $S$ (denoted by $\geq_{S}$ ) if $M\left(x^{\prime}\right) \geq_{S} M(x)$, when $x^{\prime} \geq_{X} x$ where $\left(X, \geq_{X}\right)$ is a partially ordered space. If the set relation for the powersets of $Y$ (i.e., the collection of all subsets of $Y$,denoted by $2^{Y}$ or $P(Y)$ ) based upon the set relation $\geq_{S}$ induces a partial order on the powersets on $2^{Y}$ (or perhaps $2^{Y} \backslash \emptyset$ ), we refer to the ascending correspondence also as an isotone correspondence. An antitone correspondence from $X$ to $2^{Y}$ (or perhaps $2^{Y} \backslash \varnothing$ )) is isotone in the dual order in its domain $X$.

To define an ascending and/or isotone correspondence, we consider the following set relations $\geq_{S}$ on the powersets $2^{Y}$ (or perhaps $\left.2^{Y} \backslash \varnothing\right) .{ }^{11}$ Smithson [75]

\footnotetext{
${ }^{11}$ Some classic references for set relations are Smithson [75] and Veinott [80]. For additional generalizations of "ascending" correspondences that prove useful in economics are found in
} 
and Veinott [80], have developed set relations for correspondences that admit isotone selections. In this paper, we focus on four set relations. Let $Y$ be a set, and $A, B \in 2^{Y}$. We define (i) the Veinott-Weak Set relation $\geq_{w}$ on $2^{Y}: A \geq_{w} B$, if for any $a \in A, b \in B$, either $a \wedge b \in B$ or $a \vee b \in A$.(ii) The Veinott-Strong Set Order $\geq_{s}$ on $2^{Y} \backslash \varnothing: A \geq_{s} B$, if for any $a \in A, b \in B, a \wedge b \in B$ and $a \vee b \in A$.(iii) The Smithson-Weak Set relation $\geq_{a s}$ on $2^{Y} \backslash \varnothing: A \geq$ as $B$ if either for any $b \in B$, there exists an $a \in A$ such that $a \geq b$; or, for any $a \in A$, there exists a $b \in B$ such that $a \geq b$. And, (iv) the Pointwise Strong Set Order $\geq_{s s}$ on $2^{Y}: A \geq_{s s} B$ iff $a \in A, b \in B$, then $a \geq b$ in the partial order structure on $A$, for all $a, b .{ }^{12}$ Notice that if $X$ is a lattice with $P(X) \backslash \varnothing$ ordered with induced set order implied by Veinott's strong set order $\geq_{s}$ on $X,\left(P(X) \backslash \varnothing, \geq_{s}\right)$ is a partially ordered set (actually, a lattice). Also, notice that the Veinott's strong set order is compatible with correspondences that are singleton (i.e., the standard definition of a isotone function)

Finally, a sequence $\left\{h_{n} \rightarrow h\right\}$ in $H$ is order convergent on sequences if there exists two monotonic sequences of elements from $H$, one decreasing $\left\{h_{\downarrow}\right\}$, and one increasing $\left\{h_{\uparrow n}\right\}$, such that $h=\inf h_{\downarrow n}=\sup h_{\uparrow n}$ and $h_{\uparrow n} \leq h_{n} \leq h_{\downarrow n}$. A necessary and sufficient condition for an increasing sequence $h_{n} \rightarrow h$ to be order convergent is $h=\sup h_{n}$. An operator $A h$ is order continuous on sequences on $H$ if whenever $h_{n} \rightarrow h$, in order, $A h_{n} \rightarrow A h$, in order. For example, an operator $A$ is order continuous along directed sequences $\left\{h_{n}\right\}$ if $\vee A\left(h_{n}\right)=A\left(\vee h_{n}\right)$ (see Davey and Priestley [27], chapters 2 and 3 for a discussion of order continuity and related notions).

With this investment in terminology, we are now ready to study the equilibrium problem.

\subsection{Existence and Computation of Isotone MEDPs}

Appealing to standard arguments, the Euler inequalities associated with the right side of the Bellman equation (4) can be written for each agent $j \in \mathbf{J}$ as follows,

$$
\begin{aligned}
u_{j}^{\prime}\left(c_{j}\left(s_{j}\right)\right) & \geq \beta_{j} \int_{\Theta} u_{j}^{\prime}\left(c_{j}\left(s_{j}^{\prime}\right)\right) \bar{r}\left(h(K, \theta), \theta_{a}^{\prime}\right) \chi\left(\theta, d \theta^{\prime}\right), \\
& =\text { if } k_{j}^{\prime}>0
\end{aligned}
$$

where $s_{j}^{\prime}, \theta_{a}^{\prime}$ and $k_{j}^{\prime}$ refer to next period's state, aggregate shock and capital, respectively; and $u_{j}^{\prime}$ is the marginal utility (for agent $j$ ). In a recursive competitive equilibrium, a candidate consumption function for the household of type $j \in \mathbf{J}$ necessarily satisfies $c_{j}\left(s_{j}\right)=C_{j}(S)$ pointwise when $k=K$ where $C_{j}(S)$ depends

Heikkila and Reffett [37][38].

${ }^{12}$ The $S$-weak set relation in (iii) was studied extensively in Smithson [75]. It has been subsequently referred to under the name "weak induced set order" and is characterized in Topkis ([78], p38). For a set $Y$, the weak induced set relation does not induce a valid partial order on the powersets of $Y$ (i.e., the collection $2^{Y}$ ). 
only on the aggregate state. Further, recall the income process for household $j \in \mathbf{J}$ is given as $g_{j}\left(K_{j}, K, \theta\right)=g_{j}(K, \theta)$ when $k=K$.

Given these equilibrium restrictions, we can rewrite the set of $J$ Euler inequalities in (5), which are written in terms of household consumption, equivalently, as a system of $J$ Euler inequalities in equilibrium written in terms of i investment. Given the concavity of each agent's value function in $k_{j}$ under Assumptions One-Three, a solution to the following system of equilibrium Euler system of inequalities is necessary and sufficient condition for the existence of an MEDP; namely finding a vector of investment functions $i^{*}=i^{*}(K, \theta)$, such that the consumption vector, $C=g-i^{*}>0$, when $K \geq 0$ and $K \neq 0$, in the expression,

$$
\begin{aligned}
u_{j}^{\prime}\left(g_{j}-i_{j}^{*}\right) & \left.\geq \beta \int_{\Theta} u_{j}^{\prime}\left(g_{j}\left(i^{*}, \theta^{\prime}\right)-i_{j}^{*}\left(i^{*}, \theta^{\prime}\right)\right) \bar{r}\left(i^{*}, \theta_{a}^{\prime}\right)\right) \chi\left(\theta, d \theta^{\prime}\right), \\
& =\quad \text { if } i_{j}^{*}>0 \text { for } j=1, \ldots, J
\end{aligned}
$$

where $g-i^{*}=\left[g_{1}-i_{1}^{*}, \ldots, g_{J}-i_{J}^{*}\right]$ and $g_{j}=g_{j}(K, \theta)$.

We now prove the existence of a complete lattice of functions $i^{*}(K, \theta)$ that satisfy (6). To do this, we use an equilibrium version of the system of Euler inequalities in (6) to define implicitly a nonlinear operator $A h$ on the space of candidate equilibrium investment decisions $\mathbf{H} \subset \mathbf{\mathbf { H }}$ for which a fixed point of $A h$, say $h^{*} \in \mathbf{H}$, can be shown to be an MEDP. Recalling that the space $\mathbf{\mathbf { H }}$ is a subset of the space of positive continuous functions, $\mathbf{C}^{+}(\mathbf{S})$, that is socially feasible, define the closed subset $\mathbf{H} \subset \dot{\mathbf{H}}$ of candidate equilibrium investment equilibrium functions (that have stronger isotonicity properties) as follows:

(i) $h=\left[h_{1}, \ldots, h_{J}\right]: \mathbf{K}^{J} \times \Theta \rightarrow \mathbf{K}^{J}$ is continuous,

(ii) $h_{j} \in\left[\mathbf{0}, g_{j}\right]$ for all $(K, \theta),{ }^{13}$

(iii) $0 \leq h_{j}\left(K^{\prime}, \theta\right)-h_{j}(K, \theta) \leq g_{j}\left(K^{\prime}, \theta\right)-g_{j}(K, \theta)$ for all $j$ when $K^{\prime} \geq K$ for each $\theta \in \Theta$.

We prove now prove the existence of MEDPs within this subset of candidate equilibrium investment functions. Notice the space $\mathbf{H}$ consists of continuous functions such that consumption and savings for each agent $j \in \mathbf{J}$, are both isotone in distribution of wealth for the aggregate economy (condition (iii) implies that). We remark that under Assumptions Two and Three, as $g_{j}$ is a continuous and differentiable function for all $K \geq 0, K \neq 0$, each $h_{j}$ for $j \in \mathbf{J}$ defined on a compact set $\mathbf{S}, g_{j}$ is locally Lipschitz for each agent $j$ (and, therefore, Clarke differentiable) on the interior of $\mathbf{K}^{J}$. This implies each element of $h \in \mathbf{H}$ inherits this local Lipschitz property. ${ }^{14}$

In Lemma One, as $\mathbf{H}$ is an equicontinuous subset of functions in the normal and solid cone of continuous functions $\mathbf{C}^{+}(\mathbf{S})$ when ordered by the pointwise

\footnotetext{
${ }^{13}$ We note that this restriction under Assumption Three(ii) implies $h(0, \theta)=0$ for each $\theta \in \Theta$.

${ }^{14} \mathrm{By}$ proving equilibrium of MEDPs within this class, we extend a result concerning Clarke differentiability of equilibrium policies functions due to Montrucchio [60] to our environment with many agents, production nonconvexities, and incomplete markets.
} 
Euclidean order induced by the cone, then by Arzela-Ascoli's theorem $\mathbf{H}$ is relatively compact in $\mathbf{C}^{+}(\mathbf{S})$. As $\mathbf{H}$ is also closed in $\mathbf{C}^{+}(\mathbf{S}), \mathbf{H}$ is therefore compact in $\mathbf{C}^{+}(\mathbf{S})$. As $\mathbf{H}$ is compact in a topology finer than the interval topology and a sublattice of $\mathbf{C}^{+}(\mathbf{S})$, by Frink's theorem (e.g., see Birkhoff [16], Theorem 20, page 250) we concluded $\mathbf{H}$ is subcomplete in the relative order on $\mathbf{C}^{+}(\mathbf{S})$. We summarize these observations in Lemma One. More specifically, recall $\mathbf{C}^{+}(\mathbf{S})$ is the cone of positive continuous maps $h: S \rightarrow R_{+}^{J}$ endowed with the standard $C^{0}$ uniform topology and the pointwise partial order induced by the cone. ${ }^{15}$

Lemma $1 \mathbf{H}$ is a convex, complete sublattice of the Banach lattice of continuous functions $\mathbf{C}^{+}(\mathbf{S})$. $\quad \mathbf{H}$ is also a compact order interval of continuous functions in $\mathbf{C}^{+}(\mathbf{S})$ that are locally Lipschitz on the interior of $\mathbf{K}_{+} \times \Theta$.

Let $u^{\prime}(g-i)=\left[u_{1}^{\prime}\left(g_{1}-i_{1}\right), \ldots, u_{J}^{\prime}\left(g_{J}-i_{J}\right)\right]^{T}$ denote the vector of marginal utilities. Then the $J$ Euler equations in (6) can be written more compactly as,

$$
\left.u^{\prime}(g-i)=\beta \int_{\Theta} u^{\prime}\left(g\left(i, \theta^{\prime}\right)-i\left(i, \theta^{\prime}\right)\right) \bar{r}\left(i, \theta^{\prime}\right)\right) \chi\left(\theta, d \theta^{\prime}\right) .
$$

As, in general, $u^{\prime}(c)$ is extended real valued for $c \in \mathbf{K}^{J}$, define (using the obvious vector notation) the extended real valued mapping $Z: \mathbf{K}^{J} \times \mathbf{K}_{+}^{J} \times \mathbf{H} \backslash \mathbf{g} \times \boldsymbol{\Theta} \rightarrow$ $\overline{\mathbf{R}}^{J}=\mathbf{R}^{J}+\{-\infty, \infty\},{ }^{16}$

$$
\begin{aligned}
Z(x, K, h, \theta) & =\Psi_{2}(x, K, \theta)-\Psi_{1}(x, K, h, \theta), \\
\Psi_{1}(x, K, h, \theta) & =\beta \int_{\Theta} u^{\prime}\left(g\left(x, \theta^{\prime}\right)-h\left(x, \theta^{\prime}\right)\right) \bar{r}\left(x, \theta^{\prime}\right) \chi\left(\theta, d \theta^{\prime}\right), \\
\Psi_{2}(x, K, \theta) & =u^{\prime}(g(K, \theta)-x) .
\end{aligned}
$$

Further define the nonlinear operator $A: \mathbf{H} \rightarrow \mathbf{H}^{\prime}$ implicitly in $Z$ as follows (here let $\mathbf{H}^{\prime}$ be a subset of the set of space of positive bounded functions on $\mathbf{K} \times \Theta$ ):

$$
\begin{aligned}
A h_{j}(K, \theta)= & \left\{x_{j}(K, h, \theta) \in x_{Z j}^{*}(K, h, \theta):(K, h, \theta) \in \mathbf{K} \times \mathbf{H} \times \mathbf{\Theta} ;\right. \\
& x_{j}(K, \theta, h) \text { isotone in }(K, h),
\end{aligned}
$$

where the correspondence $x_{Z j}^{*}(K, h, \theta)$ is given on $\mathbf{K}_{+}^{J} \times \mathbf{H} \backslash \mathbf{g} \times \mathbf{\Theta}$ as,

$$
x_{Z j}^{*}(K, h, \theta)=\left\{x \mid Z(x, K, h, \theta) \leq 0, \text { and } Z_{j}(x, K, h, \theta)=0 \text { if } x_{j}>0\right\} .
$$

and extended to all of $\mathbf{K} \times \mathbf{H} \times \mathbf{\Theta}$ as $x_{Z j}^{*}(K, h, \theta)=g_{j}(K, \theta)$ if $h_{j}(K, \theta)=g_{j}$ for $(K, \theta) \in \mathbf{K}^{J} \times \Theta, x_{Z j}^{*}(K, h, \theta)=0$ if $\left.K=\mathbf{0}, \theta \in \Theta\right\}$.

\footnotetext{
${ }^{15}$ Proofs of the lemmata, propositions, and theorems are in the Appendix.

${ }^{16}$ Given that $Z$ is extended real valued, notice we are careful to avoid any comparison of $\infty$ and $-\infty$ in the definition of $Z$. That is, we only define the correspondence $x_{Z j}^{*}(K, h, \theta)$ using the equation $Z$ only at points where this comparison is not made.
} 
Recalling that a compact operator is an operator that maps bounded subsets into relatively compact subsets, and a completely continuous operator is a compact operator that is also continuous in its relevant topology, we can now prove a key proposition useful in the subsequent proofs of the main theorems of the paper. ${ }^{17}$

Proposition 2 Under Assumption One-Three, (i) for any $h \in \mathbf{H}$ and $(K, \theta) \in$ $\mathbf{S}, A h(K, \theta): \mathbf{H} \rightarrow \mathbf{H}^{\prime}$ is well-defined; (ii) Ah(K, $) \subset \mathbf{H} \subset \mathbf{H}^{\prime}$; (iii) Ah is a continuous and compact operator (e.g., completely continuous) on $\mathbf{H}$ in the uniform topology on $\mathbf{H}$; (iv) Ah is isotone on $\mathbf{H}$; (v) Ah is order-continuous along sequences in $\mathbf{H}$.

Since $\mathbf{H}$ is a nonempty, compact and convex order interval in the space of continuous functions on $\mathbf{K} \times \Theta$, and $A$ is a completely continuous operator, a simple topological argument for the existence of Markovian equilibrium might be available. For example, Schauder's theorem guarantees that existence of a fixed point for such an $A$ (see, Hutson and Pym [41], page 208, Theorem 8.2.3.). In our case, this approach is not useful from the point of establishing existence of equilibrium since we already know $A$ has a trivial fixed point (namely the zero consumption plan), and in order to get interior fixed points, it proves difficult to associate a (strictly concave) value function.

An alternative approach is to follow Becker and Zilcha [8] and Miao [55]. In their approach, one defines a Markov (or adjoint) operator on the space of probability measures on $\mathbf{S}$ that aggregate the optimal policy functions of each agent $j \in \mathbf{J}$, where the policy functions are given as the solution to each agents (strictly concave) dynamic program. Given that $\mathbf{S}$ is compact, the space of probability measures is weakly compact; assuming a Feller property for the shocks in $\chi$ and noting the strict concavity to the agent dynamic program in (4), this Markov operator is weakly continuous. Existence then follows from a standard application of a local convexity fixed point (e.g. Schauder's theorem, or Fan-Glicksberg). Although this latter approach is very powerful for existence, it is unfortunately very difficult to relate such an approach to applied work using numerical methods based on constructing policy functions. Therefore, we also do not follow this approach.

We follow instead an approach that is somewhat in the spirit of Coleman [19]. The implementation of such an approach in our multiagent setting (relative to Coleman's representative agent setting) is much more complicated. First, we have equilibrium inequality constraints. Proposition 2 shows that one can systematically deal with these considerations in a class of nonlinear fixed point problem associated with Euler equations. This proposition is, therefore, of independent interest for other dynamic, general equilibrium environments. A second

\footnotetext{
${ }^{17}$ We can relate Proposition 2(i) to the generalized version of an implicit function theorem in Robinson [66]. The proof uses a Karash-Kuhn-Tucker approach to obtaining a locally Lipschitz selection. Estimates of the Clarke-gradient of this selection can be constructed directly using Robinson's results. Additional related generalizations of Robinson's arguments are discussed in the monograph by Rockafellar and Wets [65].
} 
consideration is that our operator $A h$ has a much more complicated geometric structure than the operator in a single agent economy with no borrowing constraints (and strict interiority). As in Coleman [19], we are able to exploit the monotonicity of $A$ to construct fixed points; also like Coleman [19], we are able to combine this underlying monotonicity with the results on order continuity for our operator (discussed in Proposition 2(iv)) and to apply a strong version of Tarski's theorem for the computation of extremal fixed point via successive approximation off the endpoints of the space: however, unlike Coleman [19][21] and Datta et al [24], the sufficient conditions under which our system of nonlinear operators $A h$ is strong-sublinear (or "pseudo-concave") are very strong. We defer a discussion of this issue to section five, where we make explicit how nonlinear operators in heterogeneous agent models differ radically from their homogeneous agent counterparts.

For existence and computation of MEDPs, we consider the application of the Knaster-Tarski fixed-point theorem to the operator $A$ relative to the space H.We denote the fixed points of the operator $A h$ by $E(\pi)$, and, as we are interested in how this equilibrium correspondence changes with perturbations of $\pi$, we make explicit the dependence of $E$ on the policy distortions $\pi$. The application of the Knaster-Tarski theorem is due to Kantorovich (for a proof see, for example, Vulikh [81], Lemma XXII.2.1, page 337). The theorem states that, for a isotone operator $A$ that is order continuous on sequences, mapping a nonempty complete lattice $\mathbf{H}$ into itself, for which there exists some element $\bar{h} \in \mathbf{H}$ with the property $A \bar{h} \leq \bar{h}$, has a nonempty set of fixed points. In addition, the sequence $\left\{A^{n} \bar{h}\right\}$ converges, by successive approximation, to the maximal fixed point $h_{u}^{*}=\sup E(\pi) \cdot{ }^{18}$ Using a dual argument, we can obtain similar results for the minimal fixed point $h_{l}^{*}$. In our problem, $h_{u}^{*}=g$ is a (trivial) maximal fixed point, and can therefore not be decentralized as a competitive equilibrium. Hence, we focus on the minimal fixed point. As long as $h_{l}^{*} \in E(\pi)$ is such that $g-h_{l}^{*}>>0$, then $h_{l}^{*}$ is an MEDP.

Theorem 3 Under Assumptions One-Three for any given $\pi \in \Pi$, (i) the set of fixed points of Ah, namely $E(\pi)$, is nonempty complete lattice such that any $h^{*} \in E(\pi)$ with $g-h>0$ is a MEDP; (ii) among the set of fixed points of $A$, there exists a maximal (and, respectively, a minimal) fixed point $h_{u}^{*}(\pi)$ $=\sup \mathbf{E}(\boldsymbol{\pi})$ (respectively, $h_{l}^{*}(\pi)=\inf E(\pi)$ ) such that $\lim _{n} A^{n}(g)=h_{u}^{*}(\pi)(=g)$ (respectively, $\lim _{n \rightarrow \infty} A^{n}(0)=h_{l}^{*}(\pi) \geq 0$ ) and the convergence is uniform; (iii) the minimal fixed point $h_{l}^{*}(\pi)=\inf E(\pi)$ is such that for all $j \in J, g_{j}-h_{j l}^{*}(\pi)>$ 0 ; (iv) $E\left(\pi_{k}\right)$ is isotone on $\left(\Pi_{k}, \leq_{d}\right)$ in the strong set order $\geq_{s}$ in $\pi_{k}$, where $\Pi_{k}$ $=\left\{\pi_{k} \mid\left(\pi_{k}, \pi_{n}\right) \in \Pi\right\}$ and $\leq_{d}$ is the dual standard pointwise partial order on $\Pi_{k}$, and $\sup E(\pi)$ and $\inf E(\pi)$ form isotone selections.

Our work can be related to the findings on determinacy of equilibrium of

\footnotetext{
${ }^{18}$ Note that, there are other fixed point arguments that deliver this successive approximation result. For excellent discussions, see Amann [3] and Heikkila and Lakshmikantham [36], chapter 1.
} 
Kehoe, Romer, and Woodford [47] and Santos [68]. A so-called Negishi problem is constructed, which is a modified planning problem that can be used to characterize the equilibrium manifold, and a sufficient condition for establishing comparative analysis results is that the value function be $C^{2} \cdot{ }^{19}$ In contrast, we take an order based approach to comparative analysis, and consider the question of global uniqueness within the class of equicontinuous Markovian equilibrium where both investment and consumption decisions are monotone.

\section{On the Stability of Successive Approximation Algorithms}

When applying numerical methods to the models that satisfy Assumptions OneThree, the applied researcher first develops an approximation scheme (e.g., a set of basis functions for a standard projection method a la Krasnoselskii et al ( [48], chapters 1 and 4)) that delivers asymptotically consistent numerical approximations for an unknown element of the equicontinuous collection functions $h \in \mathbf{H}$, using successive approximations on an isotone operator. Therefore, when applying such a scheme to the operator $A h$, one obtains an iterative projection method is in Kurpel [54], chapter 1). ${ }^{20}$ The existence of such approximation schemes for the class $\mathbf{H}$ is well-known. In this section, our concern is the stability of successive approximations on $A h$ from initial $h_{0} \in \mathbf{H}$.

To address the issue of stability of successive approximations, we develop sufficient conditions under which we can characterize arbitrary trajectories of iterative procedures based upon the operator $A$. In Theorem 3 , we provide a characterization concerning the computation of extremal fixed points. That is, the theorem proves that the limit of monotone iterations, $\lim _{n} A^{n}(0)$, is stable for the minimal fixed point. In this section, we identify additional conditions that sharpen the stability property of $A h$ from any initial $h_{0} \in \mathbf{H}, h_{0}<g$. That is, we characterize the $\operatorname{limit}_{1} \lim _{n} A^{n}\left(h_{0}\right)$, for any $h_{0} \in \mathbf{H}, h_{0}<g$. For this, we require an additional interiority condition, namely $A(0)>0$. We refer to this as the"strong" interiority case.

In Theorem 4, we provide a characterization of strictly interior Markovian equilibrium within the class $\mathbf{H}$. We then address the issue of uniqueness and stability of monotone iterative procedures based upon $A h$ within the class $\mathbf{H}$, and note that an implication of our argument, in Theorem 4, for the case of homogeneous agents (allowing for distortions) (e.g., Coleman [19][21], Datta et al [24],

\footnotetext{
${ }^{19}$ Santos [68] provides a set of sufficient conditions for smooth equilbrium policy functions.

${ }^{20}$ That is, we assume that the basis elements are chosen such that there exists a sequence of approximations $\hat{h}_{n} \in \mathbf{H}_{n}$ such that $\hat{h}_{n} \rightarrow h \in \mathbf{H}$, uniformly as $n \rightarrow \infty, n \in \boldsymbol{N}$, where $\boldsymbol{N}$ the set of natural numbers. The nonlinear fixed point operator $A$ defined in (7), is both topologically and order continuous. Relative to topological continuity, in particular, the trajectory $\left\{A^{n}(0)\right\}$ forms an equicontinuous seequence in $\mathbf{H}$. Therefore, an iterative projection method based upon $A \hat{h}_{n} \rightarrow A h$, for suitable $\mathbf{H}_{n}$ (e.g., piecewise linear as in Coleman [18]) has the desired convergence properties from an initial function $h_{n 0}=0$. Asymptotic convergence issues for successive approximations of extremal fixed points are, thus, straightforward, and can be addressed by various approaches, e. g., Judd (e.g, [42]).
} 
and Morand and Reffett [62]), we can greatly sharpen the sufficient conditions for uniqueness using monotone-map methods. That is, the strong monotonicity requirement proposed in Coleman [19], referred to as " $k_{0}$-monotonicity" is redundant in the setting of fixed point theorems on compact subsets of a solid cone. That is, we develop a new proof of uniqueness within the class $\mathbf{H}$ under the stated hypotheses using the following arguments in Guo and Lakshmikantham [35]: (i) a strongly sublinear operator on a solid cone is a e-concave operator ([35], Theorem 2.2.1); (ii) an isotone e-concave operator on a solid cone has at most one strictly positive fixed point ([35], Theorem 2.2.2), and (iii) a increasing completely continuous e-concave mapping $A$ has a strictly positive fixed point if and only if $A$ is a cone compression ([35], p65). ${ }^{21}$ Note that a strictly sublinear operator when the underlying domain is a solid cone is exactly what Coleman [19][21] and Datta et. al [24] refer to as a "pseudo-concave" operator. We discuss this issue more after we present our theorem. We include a proof with discussion (as we make reference to the arguments immediately following the theorem).

Theorem 4 Under Assumptions One-Three, for $\pi \in \Pi, h_{0}<g, h_{0} \in \mathbf{H}$, (i) A is a completely continuous, isotone, and e-concave operator with a fixed point correspondence $E(\pi)$ having a strictly positive element if and only if $A$ is a cone compression. Further, (ii) if $A(0)>0$, then $\min E(\pi)=h_{l}^{*}=\lim _{n} A^{n} h_{0}$ $>0$ is the unique strictly interior Markovian equilibrium within the class of equicontinuous Markovian equilibrium in $\mathbf{H}$, i.e., $h_{l}^{*}$ is a globally stable fixed point of Ah relative to the set $\mathbf{H} \backslash g$.

Proof: To prove the proposition, we first consider rewriting the operator $A h$ as a new operator $\widehat{A} m$ that can be studied more readily using geometric arguments. To do this, define the space $\mathbf{M}$ of functions $m: S \rightarrow R_{+}^{J}$ satisfying the following conditions:

(i) $m(K, \theta)=\left[m_{1}(K, \theta), \ldots, m_{J}(K, \theta)\right]$ with $m_{j}: \overline{\mathbb{K}}^{J} \times \boldsymbol{\Theta} \rightarrow \overline{\mathbb{K}}^{J}$ continuous for all $j$;

(ii) for $K>0,0 \leq m_{j}(K, \theta) \leq \frac{1}{u_{j}^{\prime}\left(g_{i}(K, K, \theta)\right)}=m^{u}$ for all $j$;

(iii) $m_{j}(0, \theta)=0$ for all $j$ and all $\theta$;

(iv) $\frac{\bar{r}\left(K^{\prime}, \theta\right)}{m_{j}\left(K^{\prime} \theta\right)} \leq \frac{\bar{r}(K, \theta)}{m_{j}(K, \theta)}$ when $K^{\prime} \geq K$.

For any $m \in \mathbf{M}$, we define the function $H(m, K, \theta)=\left[H_{1}(m, K, \theta), \ldots, H_{J}(m, K, \theta)\right]$

\footnotetext{
${ }^{21}$ Let $\mathbf{P}$ be a solid cone. An operator $\hat{A}: \mathbf{P} \rightarrow \mathbf{P}$ is strongly sublinear if for a strictly interior $m \in \mathbf{P}$, and for any $t \in(0,1)$ we have $\hat{A} t m>t \hat{A} m$ for all $(K, \theta)$. Notice a strongly sublinear operator is a pseudo-concave operator in Coleman [19] restricted to a subset of a solid cone.

An operator $A h: \mathbf{P} \rightarrow \mathbf{P}$ is a cone compression on the normal cone $\mathbf{P}$ if their exists an $R, r>0$ such that

$$
\begin{aligned}
& A h \leq h, \text { for } h \in \mathbf{P},\|h\|<r, h \neq 0 \\
& A h \geq(1+\epsilon) h, \text { for } h \in \mathbf{P},\|h\|>R, \epsilon>0 .
\end{aligned}
$$
}


implicitly in the following system of $n$ equations:

$$
\begin{aligned}
u^{\prime}\left(H_{j}(m(K, \theta))\right) & =\frac{1}{m_{j}(K, \theta)} \text { for } m>0 \\
H_{j}(m, K, \theta) & =0 \text { for } m_{j}=0 .
\end{aligned}
$$

which under our concavity, continuity, and monotonicity assumptions embodied in Assumptions One-Three can be solved globally for a unique inverse (using the inverse function theorem along with the fact that all mappings are proper). Notice, this mapping $H_{j}$ can be defined if a particular agent $j$ is borrowing constrained, i.e., $H_{j}=g_{j}$.

To begin our argument, first note that if $A(0)>0$ (which implies all agents invest in equilibrium), defining $m^{u}=\sup \mathbf{M}$, we must have $A m^{u}<m^{u}$. Therefore, the indicator variables for investment for $I_{x}(x=0)=\mathbf{0}$ in the definition of $A h$ in (7). Therefore under this condition, we can use (6) to define the operator $A h$ in (8).

Now, under the strict concavity and boundary assumptions on $u_{i}(c)$ in Assumption One, by a standard inverse function argument relative to the definition of $H$ defined above in (9), we have $H$ well defined for each $m(K, \theta) \in \mathbf{M}$. In particular, using the definition of $A h$ (which is a monotone selection in the correspondence $x^{*}(t)$ as discussed in the proof of Proposition 2 in the appendix), noting that "today's" consumption in (6) is now defined using $H=g-A h$. Further noting that "tomorrow's" consumption in (6) is $H^{\prime}=g-h$, we can rewrite $\Psi_{1}$ and $\Psi_{2}$ in (6) using (9) as follows:

$$
\begin{aligned}
\Psi_{1} & =\beta \int_{\Theta} u^{\prime}\left(g\left(x, \theta^{\prime}\right)-h\left(x, \theta^{\prime}\right)\right) \bar{r}\left(x, \theta^{\prime}\right) \chi\left(\theta, d \theta^{\prime}\right) \\
& =\beta \int_{\Theta} \frac{\bar{r}\left(g-H(\tilde{m}(K, \theta)), \theta^{\prime}\right)}{m\left(g-H(\tilde{m}(K, \theta)), \theta^{\prime}\right)} \chi\left(\theta, d \theta^{\prime}\right), \\
\Psi_{2} & =\frac{1}{\tilde{m}} .
\end{aligned}
$$

$Z(x, K, \theta, h)$ in $(6)$ then becomes with equality in all states):

$$
\hat{Z}(m, x, K, \theta)=\frac{1}{x}-\beta \int_{\Theta} \frac{\bar{r}\left(g-H\left(x, \theta^{\prime}\right)\right.}{m\left(g-H(x), \theta^{\prime}\right)} \chi\left(\theta, d \theta^{\prime}\right),
$$

where the new operator $\widehat{A}$ is defined implicitly as follows:

$$
\begin{aligned}
\hat{A} m(K, \theta) & =\left\{x(K, \theta) \in x^{*}(K, \theta) \mid \hat{Z}\left(m, x^{*}(K, \theta, K, \theta)=0,\right.\right. \\
x(K, \theta) \text { isotone, } K & \neq \mathbf{0}, m>0 ; 0 \text { elsewhere }\} .
\end{aligned}
$$

Noting (10), observe must have $\hat{A} m=x(K, \theta)=\frac{1}{u^{\prime}(g-A h)}$ for the isotone selection in (6) used to define $A h$ for this case when $h<g$. Further under Assumptions One-Three, noting $m \in \mathbf{M}$, using an argument similar to the one in the 
proof of Proposition 2, we have $\hat{Z}$ is strictly increasing in $m$, and strictly decreasing in $x$. In addition, for fixed $m>0, K \neq \mathbf{0}$ and $\theta, \hat{A} m=\frac{1}{u^{\prime}(g(K, \theta)-A h(K, \theta))} \rightarrow 0$ implies that $\hat{Z} \rightarrow+\infty$, and $\hat{A} m \rightarrow \frac{1}{u^{\prime}(g(K, \theta))}$, implies that $\hat{Z} \rightarrow \infty$. Consequently, again following arguments in Proposition 2, $\hat{A} m$ is well defined using an isotone selection in the correspondence $x^{*}(K, \theta)$ for each $m>0, K \neq \mathbf{0}$, and $\theta$. Note that $\hat{A} m=0$, elsewhere.

The operator $\widehat{A}$ is related to the mapping $B h=g-A h$ in the following manner (where for the moment, we write the operator constructed in the Proposition in terms of consumption). ${ }^{22}$ To any orbit of the operator $B h=g-A h$ (i.e., any trajectory $B^{n} h_{0}$ for $h_{0} \in \mathbf{H}$ with proposition) corresponds to an orbit of the operator $\widehat{A}$, and can be obtained through the following construction. Given any $h_{0} \in \mathbf{H}$, we have $m=\frac{1}{u^{\prime}(g-h)} \in \mathbf{M}$. (Equivalently, $m$ satisfies $H(m)=g-h$ ). Then by construction, there exists an $\widehat{A} m$ associated with any $A h$ that satisfies $\widehat{Z}\left(m_{0}, \widehat{A} m_{0}, K, \theta\right)=0$, that is,

$$
\frac{1}{\widehat{A} m(K, \theta)}=\beta E_{\theta} \frac{\bar{r}\left(g(K, \theta)-H(\widehat{A} m(K, \theta)), \theta^{\prime}\right)}{m\left(g(K, \theta)-H(\widehat{A} m(K, \theta)), \theta^{\prime}\right)} ;
$$

Therefore by the definition of $\widehat{A} m$ it must be that $1 / \widehat{A} m=u^{\prime}(B h)$ (or, equivalently, that $H(\widehat{A} m)=B h=g-A h)$. Then starting at $h_{0} \in \mathbf{H}$, by induction, we have for all $n=1,2, \ldots, B h_{0}=g-H\left(\widehat{A}^{n} m\right)$. It is also true that any fixed point of $B$ (and therefore $A h$ ) corresponds a fixed point of $\widehat{A}$. Indeed, for any $h^{*}$ fixed point of $A$, define $m^{*}=\frac{1}{u^{\prime}\left(g-h^{*}\right)}$ (or, equivalently, $H\left(m^{*}\right)=h^{*}$ ). By definition $h^{*}$ satisfies (using the definition that $B=g-A h$,

$$
\left.u^{\prime}\left(g-h^{*}(K, \theta)\right)=\beta E_{\theta} u^{\prime}\left(g\left(h^{*}(K, \theta), \theta^{\prime}\right)\right)-h^{*}\left(h^{*}(K, \theta), \theta^{\prime}\right)\right) \bar{r}\left(h^{*}(K, \theta), \theta^{\prime}\right),
$$

or, equivalently,

$$
1 / m^{*}(K, \theta)=\beta E_{\theta} \frac{\bar{r}\left(g(K, \theta)-H\left(m^{*}(K, \theta)\right), \theta^{\prime}\right)}{m^{*}\left(g(K, z)-H\left(m^{*}(K, \theta)\right), \theta^{\prime}\right)},
$$

using the fact that $B h=g-A h$, with $m^{*}$ a fixed point of $\widehat{A}$.

We now discuss the structure of $\hat{A} m$. First note that by an obvious modification of the arguments in Proposition 2, $\hat{A} m$ is (i) well-defined (e.g., this isotone selection on $\mathbf{M}$ exists since $\hat{Z}$ is increasing in $m$, and decreasing in $\tilde{m}$, $\hat{A} m^{\prime} \geq \hat{A} m$ so that $\widehat{A}$ is isotone), and (ii) $\hat{A} m \subset \mathbf{M}$ (also, from a proof similar to that in Proposition 2).

We now prove $\hat{A}$ is strongly sublinear operator. First notice that $\widehat{Z}$ define in equation (10) above is strictly decreasing in its second argument. Therefore, a sufficient condition for strong sublinearity is $\hat{A} t m>t \hat{A} m$ for $t \in(0,1)$ and $m$ strictly positive; i.e.,

$$
\hat{Z}(t m, t \hat{A} m, K, \theta)>\hat{Z}(t m, \hat{A} t m, K, \theta)=0,
$$

\footnotetext{
${ }^{22}$ In this proof, it is clearer, from a notational perspective, to write the operator in the proof in terms of consumption, not investment. That is why we use the notaton of $B h=g-A h$, where $A h$ is our original operator defined in (7).
} 
which is true since $m \in \mathbf{M}, m>0, K \neq \mathbf{0}, m$ such that $\frac{\bar{r}}{m}$ is decreasing in $K$

$$
\hat{Z}(t m, t \hat{A} m, K, \theta)=\frac{1}{\tilde{m}}-\beta \int_{\Theta} \frac{\bar{r}\left(g-H(t \tilde{m}), \theta^{\prime}\right)}{m\left(g-H(t \tilde{m}), \theta^{\prime}\right)} \chi\left(\theta, d \theta^{\prime}\right)>0
$$

As $\hat{A} m$ is a strongly sublinear operator on a compact subset of solid cone of positive continuous functions, $\hat{A} m$ is an e-concave operator ([35], Theorem 2.2.1). As further $\hat{A} m$ is additionally an isotone operator (in addition to being e-concave operator on a solid cone), therefore $\hat{A} m$ has at most one strictly positive fixed point ([35], Theorem 2.2.2). That proves (ii).

Recalling that an operator is completely continuous if it is both continuous and compact, by a theorem in Guo and Lakshmikantham [35], p65), as $\hat{A} m$ is an isotone completely continuous e-concave mapping on a subset of a normal cone, $\hat{A}$ has a strictly positive fixed point if and only if $A$ is a cone compression ([35], p65). then by a simple modification of a result in Coleman ([19], Theorem 5 ), as $\mathbf{K} \times \boldsymbol{\Theta}$ is compact, noting $\mathbf{M}$ is equicontinuous and closed (and therefore compact in the uniform metric topology on continuous functions), as we have $\hat{A} m_{n} \rightarrow \hat{A} m$ pointwise for all $(K, \theta)$, and by equicontinity this convergence is uniform on $\mathbf{K} \times \Theta$. Therefore $\hat{A}$ is a continuous operator on $\mathbf{M}$. That $\hat{A}$ is a compact operator follows from the fact the $\hat{A} \mathbf{M}=\{\hat{A} m \mid m \in \mathbf{M}\} \subset \mathbf{M}, \mathbf{M}$ compact. therefore $\hat{A} m$ is completely continuous. As we have already proven $\hat{A} m$ is isotone and e-concave, by the aforementioned result above, and we conclude that $\hat{A} m$ is a cone compression. This proves (i).

We have several remarks on this theorem. First in the case of CES preferences, we can work directly with the operator $A h$ defined in (7). That is, if $A(0)>0$, as $u^{\prime}(t m)=u^{\prime}(t) u^{\prime}(m)$, then as in Coleman [19] and Datta et. al [24], one can show directly $A h$ is a strongly sublinear operator on a compact subset of a solid cone. (That argument follows by a standard argument, for example, Coleman [19], Theorem 11). Second, our use of the strong isotonicity properties of strongly sublinear operator in Theorem 4 has important independent implications for uniqueness arguments in the existing monotone map literature for representative agent economies. In particular, in the case of homogeneous agent nonoptimal economies (e.g., Coleman [19][21]), our argument show can dispense with the assumption of $k_{0}-$ monotonicity. That is, a careful reading of our proof of the above result in Theorem 4 indicates that uniqueness of a strictly positive fixed point in a homogeneous agent economy such as Coleman [19] and Datta et al [24] is equivalent to their nonlinear fixed point operator being a cone compression. This is explicitly shown in a recent survey by Datta and Reffett $[23] .^{23}$

Also, note in our setting the necessity of the condition $A(0)>0$ for our operator to be a cone compression. First, consider the mapping $\hat{Z}(m, \hat{A} m, K, \theta)$ defined in (10). Say $A(0) \geq 0$. Then one can easily see that now $\hat{A} m$ is not e-concave. Consider $\hat{A} m$ when $A(0) \geq 0$ (and therefore $\hat{A} m^{u} \leq m^{u}$ with strict

\footnotetext{
${ }^{23}$ We say an operator $\hat{A}$ is said to be $K_{0}$-monotone if it is isotone and if there exists for any strictly positive fixed point $m_{1}$ of $\hat{A}$ some $K_{0}$ such that the following is true: for any $K_{1} \in\left[0, K_{0}\right]$ and any $m_{2}$ such that $m_{1} \geq m_{2}$ implies $m_{1}(K, \theta) \geq m_{2}(K, \theta)$ for all $K \geq K_{1}$.
} 
inequality for only for one $j \in J$ ). In this case, we modify the definition of $\hat{Z}$ to include the borrowing constraints as in the proof of Proposition 2 for the mapping $\hat{Z}$, and we obtain that $\hat{A} m$ is not strongly sublinear on all of $\mathbf{M}$; i.e.,

$$
\begin{aligned}
\hat{Z}(m, \hat{A} m, K, \theta) & =\frac{1}{\hat{A} m}-\beta \int_{\Theta} \frac{\bar{r}\left(g-H(\hat{A} m), \theta^{\prime}\right)}{m\left(g-H(\hat{A} m), \theta^{\prime}\right)} \chi\left(\theta, d \theta^{\prime}\right)-\phi I\left(\hat{A} m=m^{u}\right) \\
& =\frac{1}{t \hat{A} m}-\beta \int_{\Theta} \frac{\bar{r}\left(g-H(\hat{A} m), \theta^{\prime}\right)}{t m\left(g-H(\hat{A} m), \theta^{\prime}\right)} \chi\left(\theta, d \theta^{\prime}\right)-\frac{\phi I\left(\hat{A} m=m^{u}\right)}{t} \\
& =0 \\
& \leq \frac{1}{t \hat{A} m}-\beta \int_{\Theta} \frac{\bar{r}\left(g-H(\hat{A} t m), \theta^{\prime}\right)}{t m\left(g-H(\hat{A} t m), \theta^{\prime}\right)} \chi\left(\theta, d \theta^{\prime}\right)+\phi I\left(\hat{A} t m=m^{u}\right),
\end{aligned}
$$

where the last (weak) inequality follows from $\hat{A} t m \leq \hat{A} m$ even though $t m<m$. This lack of strict local isotonicity on the boundary is the result of the presence of occasionally binding equilibrium inequality constraints that affect the shape of $\hat{A}$ over the entire space $\mathbf{M}$. In previous work on uniqueness, without borrowing constraints ( e.g., the homogeneous agent economy in Coleman [21]), interiority conditions are strong enough to guarantee the needed strict form of isotonicity on or near the boundary. The borrowing constraint, therefore, implies when $A(0) \geq 0$ (and not $A(0)>0$ ), the fixed point operator $\hat{A} m$ is only sublinear, not strongly sublinear. Thus, we do not have sufficient geometric structure to guarantee globally unique fixed points for $\hat{A} m$.

We make a few final remarks on the limitations of these methods. First, for the numerical implementation of the operator $\hat{A} m$ or $A h$, one needs to develop algorithms for constructing continuous isotone selections. That we leave for future work. Second, Theorem 4 only provides the basis for pointwise strong set order comparative statics for MEDPs in $\mathbf{H}$. It is important to realize that our uniqueness argument does not rule out existence of Markov equilibrium outside the class $\mathbf{H}$ (or $\mathbf{M}$ ). Given the way selections are formed in the definition of the operator $A h$, we believe, for example, there could be other MEDPs within the class of bounded functions. So uniqueness here (even in the case of Theorem 4) is, in principle, a "weak" result.

Finally, the assumptions on the boundedeness of the state space can be relaxed, allowing for models with unbounded growth, for example, in the two sector incomplete markets economy studied by Krebs [50]. Using the methods in Morand and Reffett [62], one can use pure lattice theoretic fixed point constructions to study the operator $A h$, and all the main results hold. In future work, we will address the issues of long-run stationary Markovian equilibrium. To use order theoretic limit theory of Hopenhayn and Prescott [39] for characterizing the long-run ergodic measure for the capital stocks and idiosyncratic shocks, one requires joint monotonicity of equilibrium in endogenous states and random shocks. 


\section{Continuity of MEDPs with Non-monotonic In- come Processes}

We now discuss the question of existence and computation of Lipschitz (but not necessarily isotone) MEDPs. For this discussion, we can relax Assumption Three, and study income processes associated with general concave production processes. In this section, we develop an new approach to applying isotone recursive methods to economies that apparently do not necessarily have isotone MEDPs. For this new approach (the so-called "expanded state space" approach to isotone recursive methods), we only require that $j$-th household's income process in equilibrium $g_{j}\left(K_{j}, K, \theta\right)$ be written on an "expanded state space" of $\left(K_{j}, K, \hat{K}, \theta\right)$ in the form $g_{j}\left(K_{j}, K, \hat{K}, \theta\right)=r\left(\hat{K}, \theta_{a}\right) K_{j}+w\left(K, \theta_{a}\right) \theta_{j}+J(K, \hat{K}, \theta)$ where $g_{j}$ is isotone in $(K, \theta)$ and antitone in $\hat{K}$ where $\hat{K}$ denotes the mean capital stock. ${ }^{24}$ Intuitively, one can view this expanded state space method for studying functional equations such as (6) on the enlarged state space as breaking the effects of perturbations on the vector $K$ into an isotone part $(K)$ and an antitone part $(\hat{K})$. This allows one to find a function space (and a transformation of this space) where we can consider the existence of continuous (but not necessarily isotone) MEDPs that satisfy equilibrium system (6). When one then imposes $K=\hat{K}$, general equilibrium is restored. As these fixed points exist on spaces of mixed monotone functions on the expanded state space, the MEDP associated with $K=\hat{K}$, is not necessarily isotone in $K{ }^{25}$ Importantly, the MEDP remains continuous (and Lipschitz if each income process is assumed to be Lipshitz on the expanded state space. Therefore, the standard uniform approximation theory of Krasnoselskii et. al [48] can be applied to our isotone operator, and in principle, error bounds can be studied. We note uniform error bounds are then available for such an approximation scheme via Santos and Vigo [72] and Santos [69] (as we also have a collection of dynamic programming algorithms that can be tied to particular orbits of our resulting fixed point operator).

To begin our description of the mixed monotone method, we first state the following assumption on the primitives of the environment:

Assumption Four: The vector of distortions $\pi=\left[\pi_{k}, \pi_{n}\right]$ and $f$ are such that the distorted wage $\bar{w}$ and the distorted rental rate $\bar{r}$ are as in Assump-

\footnotetext{
${ }^{24}$ For example, in the classical production case with no public policy or production nonconvexity in social returns, assuming $f(k, n, \theta)=\theta k^{\alpha} n^{\beta}$, with $(\alpha+\beta)=1$, and $\alpha, \beta \geq 0$. With $n=1$, the income process for household $j$ on the expanded state space is given by

$$
\begin{aligned}
g_{j}\left(k_{j}, K, \hat{K}, \theta\right) & =r k_{j}+w \theta_{j} \\
& =\alpha \theta \hat{K}^{\alpha-1} \cdot k_{j}+\beta \theta K^{\alpha} \theta_{j} .
\end{aligned}
$$

Therefore, in this Cobb-Douglas production example, household income processes have a mixed-monotone structure on the expanded state space: this allows the total variation in household income to be broken into an isotone variation in $K$ and an antitone variation in $\hat{K}$. In equilibrium, of course, $K=\hat{K}$.

${ }^{25}$ We say a function $f(x, y): X \times X \rightarrow X$ is mixed-monotone, if $f$ is isotone in $x$ for each $y$, and antitone in $y$ for each $x$
} 
tion Three except instead of Assumption Three(ii), we assume $\left(\theta_{1}, \ldots, \theta_{J}\right) \subset R_{+}^{J}$ with $f, \theta$, and $\pi$ are such that the distorted income in equilibrium for the household $g_{j}\left(k_{j}, K, \theta\right)$ can be written as $g_{j}(k j, K, \hat{K}, \theta)=\theta_{j} \bar{w}\left(K, \theta_{a}\right)+\bar{r}\left(\hat{K}, \theta_{a}\right) k_{j}+$ $J(K, \hat{K}, \theta)$ where $g\left(k_{j}, K, \hat{K}, \theta\right)$ is isotone in $(K, \theta)$ when $k_{j}=K_{j}$ for each $\hat{K}$, and antitone in $\hat{K}$ for each $(K, \theta)$; and $\lim _{K, \hat{K} \rightarrow 0} g(0, K, \hat{K}, \theta)=0$.

Most production functions used in applied work in incomplete market models (with or without public policy and production nonconvexities) are consistent with Assumption Four.

To construct MEDPs, we first expand the state-space for the equilibrium system of Euler equations in (6) as in Reffett [63][64] and Mirman, Reffett, and Stachurski [59]. Under assumptions One, Two, and four, consider studying the solutions to (6) and (8) where we change the space of parameters for the fixed point mapping appropriately. Let $\hat{K} \in \mathbf{K}^{J}$. Define the following set of functions to be using in (6) and (8) (and the dynamic program (4) also) to be:

$$
\begin{aligned}
& h \in \quad \stackrel{\circ}{\mathbf{H}}=\left\{h(K, \hat{K}, \theta)=\left(h_{1}, \ldots, h_{J}\right) \mid\right. \\
& h_{j}(K, \hat{K}, \theta): \quad \mathbf{K}^{J} \times \mathbf{K}^{J} \times \mathbf{\Theta} \rightarrow \mathbf{K} \text { is continuous, isotone in } K \\
& \text { antitone in } \hat{K} ; \\
&\left.g_{j}(K, \hat{K}, \theta)-h_{j}(K, \hat{K}, \theta) \text { isotone in } K \text {, antitone in } \hat{K}\right\} .
\end{aligned}
$$

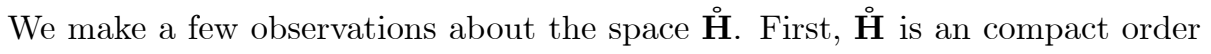
interval in the normal cone of positive continuous functions on the compact set $\mathbf{K}^{J} \times \mathbf{K}^{J} \times \boldsymbol{\Theta}$ (when the space of continuous functions on $\mathbf{K}^{J} \times \mathbf{K}^{J} \times \boldsymbol{\Theta}$ is endowed with the uniform metric topology. The cone of positive continuous functions is

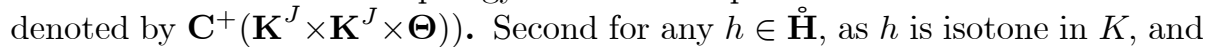

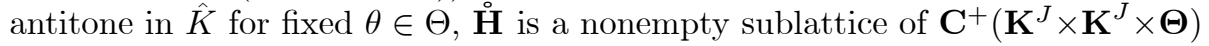
consisting of a collection of mixed-monotone functions on $\mathbf{K}^{J} \times \mathbf{K}^{J}$ for each $\theta \in \Theta$.We summarize these observations in the following lemma (the proof follows similar to lemma 1 ):

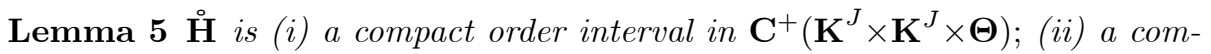
plete sublattice in $\mathbf{C}^{+}\left(\mathbf{K}^{J} \times \mathbf{K}^{J} \times \mathbf{\Theta}\right)$ under the induced partial order associated with the normal cone $\mathbf{C}^{+}\left(\mathbf{K}^{J} \times \mathbf{K}^{J} \times \mathbf{\Theta}\right)$.

Next, following the ideas of Section 4, consider the modified version of the extend-real valued mapping $Z(x, K, \theta, h)$ studied in equation $(7)$, which changes the domain of $Z$ to accommodate the expanded state space $(K, \hat{K}, \theta, h)$ used to model the mixed-monotone structure of equilibrium decision rules. Consider

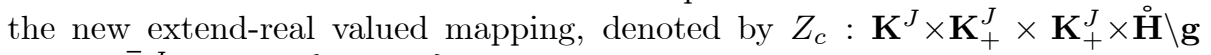
$\times \boldsymbol{\Theta} \rightarrow \overline{\mathbf{R}}^{J}=R^{n}+\{-\infty, \infty\}:$

$$
Z_{c}(x, K, \hat{K}, h, \theta)=\Psi_{2}(x, K, \hat{K}, \theta)-\Psi_{1}(x, K, \hat{K}, h, \theta),
$$


where for $j \in J$,

$$
\Psi_{1 j c}=\beta \int_{\Theta} u_{j}^{\prime}\left(g_{j}\left(X, h(K, \hat{K}, \theta), \theta^{\prime}\right)-h_{j}\left(X, h(K, \hat{K}, \theta), \theta^{\prime}\right)\right) \bar{r}\left(x, \theta^{\prime}\right) \chi\left(\theta, d \theta^{\prime}\right)
$$

and

$$
\Psi_{2 j c}=u_{j}^{\prime}\left(g_{j}\left(K, \hat{K}, \theta^{\prime}\right)-x\right) .
$$

Notice that for fixed $(\hat{K}, \theta), Z_{c}$ has the same qualitative structure in $(x, K, h)$ as $Z(x, K, h, \theta)$ (see Lemma 7 in the appendix for these results). That is, $Z_{c}(x, K, \hat{K}, h, \theta)$

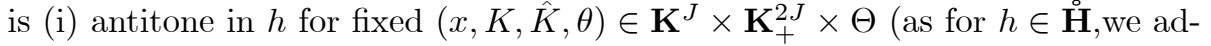
ditionally note that $g-h$ decreasing in $\hat{K}$, and therefore $\Psi_{2}$ is isotone in $h$, and $Z_{c}$ is antitone in $h$ ); (ii) strictly antitone in $K$ for each $(x, \hat{K}, h, \theta) \times \mathbf{K}^{J} \times \mathbf{K}_{+}^{J} \times$

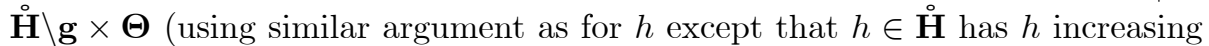
in $K$ ); and (iii) strictly isotone in $x$ for each $(K, \hat{K}, h, \theta) \in \mathbf{K}_{+}^{2 J} \times \stackrel{\mathbf{H}}{\mathbf{g}} \times \mathbf{\Theta}$. As for the comparative statics in $\hat{K}$ for each $(K, h, \theta)$, as $\hat{Z}_{c}$ has the dual structure in $\hat{K}$ as compared to $K$ by construction, $Z_{c}(x, K, \hat{K}, h, \theta)$ is isotone in $\hat{K}$ for each $(x, K, h, \theta) \in \mathbf{K}^{J} \times \mathbf{K}_{+}^{J} \times \stackrel{\circ}{\mathbf{H}} \backslash \mathbf{g} \times \mathbf{\Theta}$. Additionally, for a fixed $(x, K, h, \theta)$, it can be shown that $Z_{c}(x, K, \hat{K}, h, \theta)$ is strictly isotone in $\hat{K}$.

Define $\stackrel{\circ}{\mathbf{T}}_{+}=\mathbf{K}_{+}^{J} \times \mathbf{K}_{+}^{J} \times \stackrel{\circ}{\mathbf{H}} \backslash \mathbf{g}$ where $(K, \hat{K}, h)$ is a typical element of $\stackrel{\circ}{\mathbf{T}}_{+}$, where we give the subspace $\hat{K} \in \mathbf{K}_{+}^{J} \subset \mathbf{R}_{+}^{J}$ the dual componentwise order on

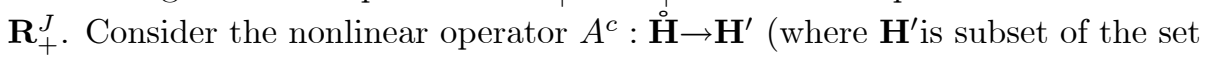
of bounded functions on $\left.\mathbf{K}^{J} \times \mathbf{K}^{J} \times \boldsymbol{\Theta}\right)$ for $j \in \mathbf{J}$,

$$
\begin{aligned}
A^{c} h_{j}(K, \hat{K}, \theta) & =x_{j}(K, \hat{K}, h, \theta) \in x_{Z j c}^{*}(K, \hat{K}, h, \theta) \text { for }(K, \hat{K}, h) \in \stackrel{\circ}{\mathbf{T}}_{+}, \\
\theta & \in \Theta, x_{j}(K, \hat{K}, \theta, h) \text { isotone in }(K, \hat{K}, h) \text { given } \theta \in \Theta,
\end{aligned}
$$

where the correspondence $x_{Z c}^{*}(K, \hat{K}, h, \theta)$ is given on $\stackrel{\circ}{\mathbf{T}}_{+} \times \boldsymbol{\Theta}$ as

$x_{Z c}^{*}(K, \hat{K}, h, \theta)=\left\{x \mid Z_{c}(x, K, \hat{K}, h, \theta) \leq 0\right.$, and $Z_{j_{c}}(x, K, \hat{K}, h, \theta)=0$ if $\left.x_{j}>0\right\}$.

and $x_{Z c}^{*}(K, h, \theta)=g_{j}(K, \hat{K}, \theta)=x_{Z j c}^{*}(K, \hat{K}, h, \theta)$ when $h_{j}(K, \hat{K}, \theta)=g_{j}$ for $(K, \hat{K}, \theta) \in \mathbf{K}^{2 J} \times \Theta$; and $x_{Z c}^{*}(K, h, \theta)=0=x_{Z j c}^{*}(K, \hat{K}, h, \theta)$ when $K$ and/or $\hat{K}=\mathbf{0}, \theta \in \Theta$ (i.e., we extend $x_{Z}^{*}(K, h, \theta)$ to all of $\left.\mathbf{K}^{J} \times \mathbf{H} \times \mathbf{\Theta}\right)$. Remember as the space for $\hat{K} \in \mathbf{K}_{+}^{J}$ is given the dual order, $A^{c} h$ is antitone in $\hat{K}$ in the standard componentwise partial order on $\mathbf{R}_{+}^{J}$. Four.

We now extend Theorem 3 to economies under Assumptions One, Two and

Theorem 6 Under Assumptions One, Two and Four, for fixed $\pi \in \Pi$,(i) the set

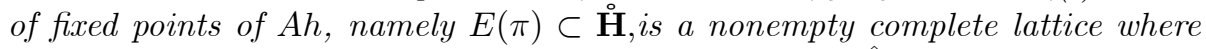
the set of MEDPs is given along the restriction $K=\hat{K}$; (ii) among the set of fixed points of $A$, there exists a maximal (respectively, minimal) fixed point $h_{u}^{*}(\pi)=\sup \mathbf{E}(\boldsymbol{\pi})\left(\right.$ resp, $\left.h_{l}^{*}(\pi)=\inf E(\pi)\right)$ such that $\lim _{n} A^{n}(g)=h_{u}^{*}(\pi)=g$ (resp, $\lim _{n \rightarrow \infty} A^{n}(0)=h_{l}^{*}(\pi) \geq 0$ ) and the convergence is uniform. (iii) the 
minimal fixed point $h_{l}^{*}(\pi)=\sup E(\pi)$ is such that at least for one index $j \in J$, $g_{j}-h_{j l}^{*}(\pi)>0$; (iv) $E\left(\pi_{k}\right)$ is isotone on $\left(\Pi_{k}, \leq_{d}\right)$ in the strong set order $\geq_{s}$ in $\pi_{k}$ where $\Pi_{k}=\left\{\pi_{k} \mid\left(\pi_{k}, \pi_{n}\right) \in \Pi\right\}$ and $\leq_{d}$ is the dual standard pointwise partial order on $\Pi_{k}$, and $\sup E(\pi)$ and inf $E(\pi)$ form isotone selections.

\section{Conclusion}

This paper develops an isotone recursive fixed point approach to the study of MEDPs in multiagent incomplete markets models with production. Our methods are based on the existence of Euler equations (which are needed to provide sharp geometric characterizations of the nonlinear operators used to construct Markovian equilibrium), and concavity of the underlying production and preference primitives. One advantage of our method is that they are developed using a single valued continuous operator that maps an equicontinuous set of functions into itself. This latter fact (concerning continuity and compactness) is critical for numerical approaches to computing the equilibrium. In particular, versions of the Stone-Weirstrass theorem can be used to prove that projection methods ala Krasnoselskii et al [48] (e.g. piecewise linear methods based upon Coleman [18] or more general projection methods based on splines and orthogonal polynomials as in Judd [42]) are asymptotically consistent. That is, as the dimension of the basis set used to approximate both the elements of the domain as well as the operator, tend toward infinity, the projection converges to the actual equilibrium fixed point.

The policies in our equilibrium set are locally Lipschitz continuous functions, and each agent's value function (in equilibrium when $k=K$ ) is concave. These are key properties that could be exploited to achieve the error bounds on numerical solutions via dynamic programming arguments, as in Santos and Vigo [72], or more general non-parametric error bound methods as presented in Santos [69]. Since trajectories of operators can be tied explicitly to a collection of value functions (one for each type of agent), one should, in principle, be able to apply Santos [69] to construct asymptotic error bounds to numerical methods based upon our monotone operator $A$ following a version of the argument in Mirman, Morand, and Reffett ([58], section 4). We leave this work for the future.

Additionally, the issue of existence of long-run stationary Markovian equilibrium remains, as do the characterization of the equilibrium pricing functional. One can change the assumptions on the primitive data of the model to obtain sufficient conditions under which Markovian equilibrium are jointly monotone in $(K, \theta)$. For example, adapting a condition studied in Mirman, Morand, and Reffett [58] to our setting, one might assume $u$ and $f$ are such that $u^{\prime \prime}(c) f_{1} f_{2}+u^{\prime}(c) f_{12} \geq 0$. In our setting, this condition is sufficient to show that the Markovian equilibrium in Theorem 3 is actually jointly monotone in $(K, \theta)$. In this case, using the limiting distribution theory of Hopenhayn and Prescott [39] and Danthine and Donaldson [26], one can construct a stationary Markovian equilibrium in the sense of Duffie et al [29]. We leave further work along this line to future research. 
In this economy, we study MEDPs in situations where the production processes are bounded. Morand and Reffett [62] show how to relax this condition for representative agent economies. A similar argument is available in this paper. If one assumes that the income processes $g_{j}\left(k_{j}, K, \theta\right)$ associated with the distorted production function $f\left(k, n, K_{m}, N, \theta\right)$ are uniformly continuous jointly in $\left(k_{j}, K\right)$

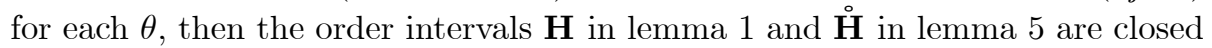
equicontinuous subsets of the space of continuous functions on the unbounded state space $\mathbf{K} \times \Theta$. Although not compact, these spaces are complete lattices. In this case, the arguments of Theorem 3 and 5 carry through with little modification. Of course, in such a setting, one must also verify that the functional equation describing agent preferences in (4) can be solved on a space of bounded value functions. But, in principle, allowing for asymptotic growth in two sector models (for example, Krebs [50]) is not a problem.

Finally, it might be possible to develop a method of monotone comparative analysis for the Markovian equilibrium set for this economy under more general assumptions on the primitive data of taste, technology, and the class of equilibrium distortions considered. In many situations concavity of the value function is lost, and, therefore, single valued operators on spaces of continuous functions are no longer available. However, it is often possible to apply generalizations of Tarski's theorem to such problems by exploiting the fact that equilibrium best responses (under weak supermodularity assumptions along an equilibrium path) are uniformly bounded and monotone functions. Spaces of uniformly bounded, monotone functions form complete lattices, and thus, existence and characterization using order based fixed point theory are available. Mirman, Morand, and Reffett [58] show how such methods can be used to construct monotone Markovian equilibrium for a large class of nonoptimal representative agent models. Further, using these methods, it should be possible to study with monotone methods multisector, multiagent economies with incomplete markets such as those presented in a series of papers by Krebs [50][49]. His results concern a symmetric class with identical CES preferences, constant returns to scale technologies, and no public policy. Our methods could be used to extend these results. One might develop a set of tools that would make general versions of the Krebs environment tractable for applied researchers interested in incorporating human capital and physical capital into a richer model of economic fluctuations and growth that also allow for incomplete markets and trade: perhaps, mixed-monotone methods can be used to obtain analytical results.

\section{Appendix: Proofs}

In the appendix, we discuss all the proofs of the lemmata, propositions, and theorems in the paper. We begin with the proof of Lemma 1:

Proof of Lemma 1:

Convexity and the fact that $\mathbf{H}$ is a closed sublattice of the Banach lattice of 
continuous functions when equipped with the standard pointwise partial order on $\mathbf{C}^{+}(\mathbf{S})$ is obvious. $\mathbf{H}$ is also clearly an order interval.

For compactness, simply note that $\mathbf{H}$ is an equicontinuous set of functions defined on a compact space under the restriction that $g-h$ is increasing in $K$, $\Theta$ is discrete, and $g-h$ a continuous function on a compact set (and therefore the variation in $h$ is uniformly bounded by a uniformly continuous function $g$ ). Therefore, and by a standard application of the Arzela-Ascoli theorem, $\mathbf{H}$ is relatively compact in $\mathbf{C}^{+}(\mathbf{S})$. As $\mathbf{H}$ is already closed in $\mathbf{C}^{+}(\mathbf{S}), \mathbf{H}$ is compact in $\mathbf{C}^{+}(\mathbf{S})$. As $g$ is Lipschitz in $K$ on $\mathbf{K}_{+}$and $\Theta$ is denumerable (and therefore Lipschitz in $\theta$ in the discrete topology), we therefore conclude that $\mathbf{H}$ consists of Lipschitz continuous functions on $\mathbf{K}_{+} \times \Theta$.

Finally as $\mathbf{H}$ is a closed sublattice in $\mathbf{C}^{+}(\mathbf{S})$ that is also equicontinuous in the $C^{0}$ uniform topology, $\mathbf{S}$ is compact, therefore $\mathbf{H}$ is compact in the interval topology. We then conclude $\mathbf{H}$ is a complete sublattice by the complete characterization of complete lattices in the order topology by Frink-Birkhoff Theorem ( [16], Chapter 10, Theorem 20). See also the discussion in Frink [32].)

So for any subset of $\mathbf{H}$, say $H \subset \mathbf{H}, \sup _{K, \theta} H=h^{u}(K, \theta)$ and $\inf _{K, \theta} H=$ $h^{l}(K, \theta)$ are in $\mathbf{H}$.

We next consider the proof of Proposition 2. Prior to proving this proposition, we prove two lemmata are useful. In Lemma 7 , we first note some properties of the extended-real mapping $Z(x, K, h, \theta): \mathbf{K}^{J} \times \mathbf{K}_{+}^{J} \times \mathbf{H} \backslash \mathbf{g} \times \mathbf{\Theta} \rightarrow \overline{\mathbf{R}}^{J}$ as defined in (7) of Section 4 that prove useful when characterize the mapping $x_{Z}^{*}(K, h, \theta)$ in $(7)$ on $\mathbf{K}_{+}^{J} \times \mathbf{H} \backslash \mathbf{g} \times \mathbf{\Theta}$.

Lemma 7 Under assumptions One-Three,for fixed $\theta \in \Theta$, (i) $Z(x, K, h, \theta)$ is antitone in $h$ with each $Z_{j}(x, K, h, \theta)$ is strictly decreasing in $h_{j}$; (ii) $Z(x, K, h, \theta)$ is strictly decreasing in $K$; and (iii) $Z_{j}(x, K, h, \theta)$ is strictly increasing in $x$.

Proof: (i) Fix $(x, K, \theta)$, and let $h^{\prime} \geq h\left(\right.$ for $\left.h, h^{\prime} \in \mathbf{H} \backslash \mathbf{g}\right)$. Under assumption $1, \Psi_{1}$ is increasing in $h$ (as the vector $u(c)$ is concave); therefore $Z$ is antitone in $h$.

Now, take $h_{j}^{\prime} \geq h_{j}, h^{\prime}, h \in \mathbf{H} \backslash \mathbf{g}$. Then by assumption $1, \Psi_{1 j}$ is increasing as each $u_{j}\left(c_{j}\right)$ for $j \in \mathbf{J}$ is strictly concave, therefore we have for each $Z_{j}\left(x, K, h_{j}^{\prime}, \theta\right) \leq Z_{j}\left(x, K, h_{j}, \theta\right)$; if $h_{j}^{\prime} \geq h_{j}$, and $h_{j}^{\prime} \neq h_{j}$, then the inequality is strict. Therefore $Z_{j}$ is strictly antitone in $h$.

(ii) Fix $(x, h, \theta)$, and take $K^{\prime} \geq K$. A similar argument to the above for $h$ shows the system $\Psi_{1}$ is increasing in $K$. Now, as $g$ is strictly increasing in $K$ by Assumptions Two and Three, we have $\Psi_{2}$ decreasing in $K$. So $Z$ is antitone in $K$. Again, if $K^{\prime} \neq K$, and $K^{\prime} \geq K$, all the inequalities are strict. Therefore $Z$ is strictly antitone in $K$.

(iii) For each $(K, h, \theta)$, when $x^{\prime} \geq x, \Psi_{2}$ decreases by Assumptions OneThree and the fact $h \in \mathbf{H} \backslash \mathbf{g}$, while $\Psi_{1}$ increases by Assumption One; therefore $Z\left(x^{\prime}, K, h, \theta\right) \geq Z(x, K, h, \theta)$. If $x^{\prime} \geq x, x^{\prime} \neq x$ then the inequality is strict by 
the strict concavity of $u(c)$ is assumption One and the fact that $\bar{r}$ is decreasing in Assumption Three.

Let $\left(\mathbf{X}, \geq_{x}\right)$ and $\left(\mathbf{Y}, \geq_{y}\right)$ be a complete lattices, $y^{*}(x): \mathbf{X} \rightarrow \mathbf{2}^{\mathbf{Y}} \backslash \varnothing$ a nonempty correspondence, and $X \subset \mathbf{X}$ a chain. If for any $m(x): X \rightarrow \mathbf{Y}$ be any isotone function on $X$ such that $m(x) \in y^{*}(x), x_{0}=\sup X$, we have $m\left(x_{0}\right) \leq y\left(x_{0}\right) \in y^{*}\left(x_{0}\right)$, we say the mapping $y^{*}(x)$ has the property of $m a$ jorizing chain subcompleteness (MCSC). Further if for any $x_{1} \leq x_{2}$ and $y\left(x_{1}\right) \in$ $y^{*}\left(x_{1}\right)$ and $y\left(x_{2}\right) \in y^{*}\left(x_{2}\right)$ such that $y\left(x_{1}\right) \leq y\left(x_{2}\right)$, we have for $x_{1} \leq x \leq$ $x_{2}, y^{*}(x) \cap\left[y\left(x_{1}\right), y\left(x_{2}\right)\right] \neq \varnothing$, then we say that $y^{*}(x)$ has the range intersection property $(R I P)$.

We now prove the following lemma concerning the nonempty correspondence $y(x): X \rightarrow 2^{Y} \backslash \varnothing:^{26}$

Lemma 8 Let $\left(\mathbf{X}, \geq_{X}\right)$ and $\left(\mathbf{Y}, \geq_{Y}\right)$ be complete lattices, $y^{*}(x): \mathbf{X} \rightarrow 2^{\mathbf{Y}} \backslash \varnothing$ be a nonempty and chain complete-valued for each $x \in \mathbf{X}$. Assume additionally that $y^{*}(x)$ is ascending in Smithson's weak set relation (C1) and (C2) on $\mathbf{X}$. Then $y^{*}(x)$ admits an isotone selection.

Proof: Let $\mathrm{X}$ be the collection of nonempty subsets of $2^{\mathbf{X}} \backslash \varnothing$ with typical element $X \in \mathbf{X}$ where each $X$ satisfies (i) the minimal element of $\mathbf{X}$ given as $x_{L}=\wedge \mathbf{X} \in \mathbf{X}$, and (ii) for $z \leq x \in \mathbf{X}, z \in \mathbf{X}$. Define the collection of isotone functions $g: \mathbf{X} \rightarrow \mathbf{Y} \in \mathbf{G}$ such that $g(x) \in y^{*}(x)$ for each $x \in X$. Define the following partial order on the pair $(\mathbf{X}, g) \in \mathbf{X} \times \mathbf{G}:(X, g) \leq\left(X^{\prime}, g^{\prime}\right)$ if and only if $X \subset X^{\prime}$ and $g=g^{\prime} \mid X$ (where $g^{\prime} \mid X$ denotes the restriction of $g^{\prime}$ to $X$ ). This is a valid partial order. (See for example Smithson [75], Theorem 1.9). By Zorn's lemma, there is a maximal pair, say $\left(X_{0}, g_{0}\right) \in \mathbf{X} \times \mathbf{G}$. If $X_{0}=\mathbf{X}, g_{0}(x)$ is the isotone selection.

Therefore assume $x \in \mathbf{X} \backslash X_{0}$. Define $C$ to be the maximal chain containing $x_{L}$ and $x$, and consider $C \cap X_{0}=C_{1} \neq \varnothing$ (as at least we have $x_{L} \in C_{1}$ ). Let $x_{0}=\sup C_{1}$. As $y^{*}(x)$ is ascending in both Smithson's weak set relation $(\mathrm{C} 1)$ and $(\mathrm{C} 2)$, then by the maximality of $\mathbf{X}$, we must have the following two facts: (i) $x_{0} \in X_{0}$; and (ii) there exists a $y^{\prime} \in y(x)$ such that $g_{0}\left(x_{0}\right) \leq y^{\prime}$. Consider the set $X_{1}$ where we have $X_{0} \subset X_{1}=X_{0} \cup\left\{z \mid x_{0} \leq z \leq x\right] \in \mathrm{X}$. Define mapping $\hat{g}(x): X_{1} \rightarrow \mathbf{Y}$ in the following two steps. First, let $\hat{g}(x)=g_{0}(x)$ for $x \in X_{0}$. Second to extend $\hat{g}(x)$ onto all of $X_{1}$, we first note a two facts about $y^{*}(x)$ on $X_{1}$ : (i) for $z \in\left[x_{0}, x\right]$, as $y^{*}(x)$ is antichained for each $x, y^{*}(x)$ is chain subcomplete for each $x \in\left[x_{0}, x\right]$; (ii) we can define the nonempty subset $S\left(\left[x_{0}, x\right]\right)=\cup_{x \in\left[x_{o}, x\right]} y^{*}(x) \subset \mathbf{X}$. where $S\left(\left[x_{0}, x\right]\right)$ is chain subcomplete in $\mathbf{X}$. We can now define the extension of $\hat{g}$ to all of $X_{1}$. Consider the correspondence $S(z)=y^{*}(z) \cap\left[y\left(x_{1}\right), y\left(x_{2}\right)\right] \neq \varnothing\left(\right.$ as $y^{*}(x)$ is Smithson weak ascending in both $(\mathrm{C} 1)$ and $(\mathrm{C} 2)$ ). Notice $S(z)$ is antichained valued as $y^{*}(x)$ is antichain for each

\footnotetext{
${ }^{26}$ This lemma is closely related to Veinott's isotone selection theorem [80] ([80], Chapter 4, Theorem 5) and Smithson [75], Theorem 1.9).
} 
$x \in\left[x_{0}, x\right] . S(z)$ is therefore chain subcomplete for each such $z$. As $y^{*}(x)$ is ascending in Smithson weak set relation (C1) and (C2), there exists a subchain, say $s\left[\left(x_{0}, x\right)\right] \subset S\left(\left[x_{0}, x\right]\right)$ with typical element $s(z) \in S(Z)$, such that we have the following true: (a) $s\left[\left(x_{0}, x\right)\right]$ chain subcomplete; and (b) $\wedge s\left(\left[x_{0}, x\right]\right)=\hat{g}\left(x_{0}\right)$. Therefore define the function $\hat{g}(z)=s(z) \in s\left[\left(x_{0}, x\right]\right)$ for each $z \in\left[x_{0}, x\right]$. The mapping $\hat{g}(x): X_{1} \rightarrow \mathbf{Y}$ is a well-defined isotone function on $X_{1}$. But then given the partial order on $\mathbf{X} \times \mathbf{G}$, as $X_{0} \subset X_{1}$, this implies $\left(X_{1}, \hat{g}\right)>\left(X_{0}, g_{0}\right)$. But this contradicts the maximality of $\left(X_{0}, g_{0}\right)$.

Therefore there is an isotone selection $g_{0}(x): \mathbf{X} \rightarrow \mathbf{Y}$

The operator $A h$ defined in (7) and (8) is any isotone selection in the correspondence $x_{Z}^{*}(K, h, \theta)$. We now characterize the structure of $x_{Z}^{*}(K, h, \theta)$ so as to check the conditions of Lemma 8 are satisfied for $x_{Z}^{*}(K, h, \theta)$. To do this, we first rewrite the inequalities in (8) used to define $x_{Z}^{*}$ as a collection of KarashKuhn-Tucker conditions (KKT) in equilibrium. We then define a new mapping $\hat{\Psi}(x, K, \theta, h)$ that adds to the mapping $Z(x, h, K, \theta)$ this new collection of $J$ equilibrium KKT multipliers, one for each agent who in equilibrium might be borrowing constrained in (6).

We first define the equilibrium KKT multipliers. Fix $t=(K, h) \in \mathbf{T}_{+} \stackrel{\circ}{=}$ $\mathbf{K}_{+}^{J} \times \mathbf{H} \backslash \mathbf{g}$. Recalling the definition of the mapping $Z: \mathbf{K}^{J} \times \mathbf{T}_{+} \times \boldsymbol{\Theta} \rightarrow \overline{\mathbf{R}}^{J}$, we define a new mapping $\phi(x, t, \theta): \mathbf{K}^{J} \times \mathbf{T}_{+} \times \mathbf{\Theta} \rightarrow \overline{\mathbf{R}}^{J}$ defined componentwise for each $j \in J$ as follows:

$$
\phi_{j}(x, t, \theta)=\Psi_{j 2}(x, K, \theta)-\Psi_{j 1}(x, t, \theta) \text { for } j=1,2, \ldots, J .
$$

By Lemma $7, \phi(x, t, \theta)$ is well-defined ( as the mapping $Z$ is well defined). ${ }^{27}$ Further for fixed $(t, \theta) \in \mathbf{T}_{+} \times \Theta$, we have the following properties for the vector of KKT multipliers $\phi(x, t, \theta):(\mathrm{a}) \phi$ is continuous and strictly increasing in $x$ $\in \operatorname{int}\left(\mathbf{K}^{J}\right)$, and $\phi$ is upper-semicontinuous and strictly increasing in $x$ on $\mathbf{K}^{J}$; (b) for $x=\mathbf{0}, \phi(\mathbf{0}, t, \theta)=-\infty$ (as $h \in \mathbf{H}$ and we have the Inada condition in Assumption One); and (c) $\phi_{J_{1}}\left(x_{-J_{1}}, \mathbf{g}_{J_{1}}, t, \theta\right)=\infty$ (by Assumption One for any subset of indices of agents $J_{1} \subset J$ where $x_{-J_{1}}$ is the vector $x$ with the components for agents $j \in J_{1}$ deleted).

Therefore the KKT multipliers in equilibrium are defined by the vector $\phi \cdot I_{x}(x=0)$ where the $j t h$ component of the $\operatorname{KKT}$ is given by $\phi_{j}(x, K, h, \theta)$. $I_{x_{J}}\left(x_{j}=0\right)$ where $I_{x}\left(x_{j}=0\right)$ is an indicator variable for the condition $x_{j}=0$. Define the extended-valued mapping $\hat{\Psi}$ that embeds $Z$ and the KKT multipliers $\phi I_{x}(x=0)$ allowing us to study (8) using a single system of functional inequalities: namely, consider extended real valued mapping $\hat{\Psi}(x, t, \theta)$ $: \mathbf{K}^{J} \times \mathbf{T}_{+} \times \boldsymbol{\Theta} \rightarrow \overline{\mathbf{R}}^{J}:$

\footnotetext{
${ }^{27}$ Given the definition of the operator $A h$, we do not need to consider the situation defining $A h$ when $h \in \mathbf{H}, K \in \mathbf{K}^{J}$, but $(K, h) \notin \mathbf{T}_{1}$. See the definition of the operator $A h$ in $(7)$.
} 


$$
\begin{aligned}
\hat{\Psi}(x, t, \theta) & =\Psi_{2}(x, K, \theta)-\hat{\Psi}_{1}(x, t, \theta) \text { and } \\
\hat{\Psi}_{1} & \left.=\beta \int_{\Theta} u^{\prime}\left(g\left(x, \theta^{\prime}\right)-h\left(x, \theta^{\prime}\right)\right)\right) \bar{r}\left(x, \theta^{\prime}\right) \chi\left(\theta, d \theta^{\prime}\right)+\phi(x, t, \theta) I_{x}(x=0), \\
\Psi_{2} & =u^{\prime}(g(K, \theta)-x),
\end{aligned}
$$

where $I_{x}(x=0)$ is the indicator variable on vector of equations whose components indicate in each equation $j \in \mathbf{J}$ whether household $j$ is borrowing constrained, i.e., $I_{x_{j}}=1$ when $x_{j}=0$.

Prior to proving Proposition 2, we make a few observations about the system $\hat{\Psi}(x, t, \theta)$. Under assumptions One-Three, noting the results in Lemma 7 and given the definition of $\phi$, we conclude the system $\hat{\Psi}(x, t, \theta)$ is well-defined. Further observe that the following four statements concerning the structure of the system $\hat{\Psi}$ are the case: for any fixed $(t, \theta) \in \mathbf{T}_{+}$, we have (x.i) as $x_{j} \rightarrow g_{j}(K, \theta), \hat{\Psi}_{j} \rightarrow \infty$ for any $j \in \mathbf{J} ;(x . i i)$ as $x \rightarrow g(K, \theta)$, the entire system $\hat{\Psi} \rightarrow \infty$ (both of these as $I_{x}=\mathbf{0}$, and $Z \rightarrow \infty$ ); (x.iii) letting $x=\left(x_{-j}, x_{j}\right.$ ) for some component $j \in J_{1} \subset \mathbf{J}$ where $x_{-j}$ deletes $x_{j}$ from $x$, when $x_{-j} \rightarrow \mathbf{0}_{-j}$, $x_{j}>\mathbf{0}_{j}, \hat{\Psi}_{-j}(x, t, \theta) \leq 0$; and finally (x.iv) when additionally $x_{j} \rightarrow \mathbf{0}_{j}$ (implying therefore that vector $x \rightarrow \mathbf{0}$ ), then by the definition of $\phi$ (noting Assumption Three and $h \in \mathbf{H} \backslash \mathbf{g})$, we have $\lim _{x \rightarrow \mathbf{0}} \hat{\Psi}(x, t, \theta) \leq \mathbf{0}^{28}$.

We are now ready to prove Proposition 2.

\section{Proof of Proposition 2:}

(i): The proof of this claim takes place in sequence of steps that check the hypotheses of lemma 8.

Claim A: The correspondence $x_{Z}^{*}(t, \theta): \mathbf{T} \times \Theta \rightarrow \mathbf{2}^{\mathbf{K}^{J}}$ is nonempty for each $(t, \theta) \in \mathbf{T} \times \Theta$.

Proof: We first prove $x_{Z}^{*}(t, \theta)$ is nonempty on $\mathbf{T}_{+} \times \Theta$. First note that for any $(t, \theta) \in \mathbf{T}_{+} \times \Theta$, as by Lemma 7 (iii) we have $\hat{\Psi}$ strictly increasing and continuous in $x$ on $[0, g(K, \theta))$, and nondecreasing and upper-semicontinuous on $[0, g(K, \theta)]$ with $\hat{\Psi}_{j}=0$ when $x_{j}=g_{j}$, therefore for each $(t, \theta) \in \mathbf{T}_{+} \times \Theta$ there is a pair of vectors $\left(x_{l}(t, \theta), x^{T}(t, \theta)\right) \in[0, g(K, \theta)] \times[0, g(K, \theta)]$ such that (a) $\hat{\Psi}\left(x_{l}(t, \theta), t, \theta\right) \leq 0$; (b) $\hat{\Psi}\left(x^{T}(t, \theta), t, \theta\right) \geq 0$, and (c) $x_{l}(t, \theta) \leq x^{T}(t, \theta)$ in the standard component product Euclidean order on $R^{J}$, and (iii) $x_{l}(t, \theta) \neq 0$. As $\hat{\Psi}$ is strictly increasing and continuous jointly in $x$ on $(0, g(K, \theta))$ and nondecreasing and upper-semicontinuous on all of the (connected) order interval $[0, g(K, \theta)]$, it is is nondecreasing in $x_{j}$ on $\left[0, g_{j}(K, \theta)\right]$ for fixed $x^{j}=$ $\left(x_{1}, x_{2}, \ldots x_{j-1}, x_{j+1}, \cdots, x_{J}\right), x=\left(x_{j}, x^{j}\right)$ each $j \in J$. The mapping $\hat{\Psi}(x, t, \theta)$ therefore satisfies the semi-continuity conditions in the generalized intermediate

\footnotetext{
${ }^{28}$ Notice, Assumption Three(i) is not needed for our fixed point construction. Given the definition of $\phi$, our operator can easily be defined. Assumption Three guarantees that we have non-trivial Markov equilibrium with non-zero production.
} 
value theorem on arbitrary product spaces of Guillerme ([34], Theorem 3) on $\left[x_{L}(t, \theta), x^{T}(t, \theta)\right]$. Therefore we conclude that correspondence $x_{Z}^{*}(t, \theta)$ is nonempty on the connected suborder interval $\left[x_{l}(t, \theta), x^{T}(t, \theta)\right] \subset[0, g(K, \theta)] \subset R_{+}^{J}$ with $x_{l}(t, \theta) \neq 0$. Therefore we conclude that $x_{Z}^{*}(t, \theta)$ is nonempty for each $(t, \theta) \in \mathbf{T}_{+} \times \Theta$. Notice that by the definition of the extension of $x_{Z}^{*}(t, \theta)$ in $(8)$, $x_{Z}^{*}$ is well defined for any $(t, \theta) \in \mathbf{T} \backslash \mathbf{T}_{+} \times \Theta$. Therefore $x_{Z}^{*}(t, \theta): \mathbf{T} \times \mathbf{\Theta} \rightarrow \mathbf{K}^{J}$ is well-defined. $\nabla$

Remark: Before we proceed to Claim B, we make a few additional remarks concerning some additional properties of the correspondence $x_{Z}^{*}(t, \theta)$. First, observe as $\hat{\Psi}$ strictly increasing in $x$ on $[0, g(K, \theta)]$, we have (x.vi) $x_{Z}^{*}(t, \theta)$ antichain-valued for each $(t, \theta) \in \mathbf{T}_{+} \times \Theta$ relative to the componentwise order on $R_{+}^{J}$ (Dacic [22], Proposition 1.1). ${ }^{29} \quad$ Fix $\theta \in \Theta$, and consider $x(t, \theta) \in$ $x_{Z}^{*}(t, \theta)$ for $t \in \mathbf{T}_{+}$. By the definition $x_{Z}^{*}$, for any $x(t, \theta) \in x_{Z}^{*}(t, \theta)$, we have $\hat{\Psi}(x(t, \theta), t, \theta)=0$. Consider $t^{\prime} \geq t, t \neq t^{\prime}, t$ and $t^{\prime}$ in $\mathbf{T}_{+}$. By Lemma $7(\mathrm{i})$, as $\hat{\Psi}$ is strictly antitone in $t$, we have $-\infty<\hat{z}=\hat{\Psi}\left(x(t, \theta), t^{\prime}, \theta\right)<0$. Define the directed net $G(x(t, \theta))=\{x \mid x \geq x(t, \theta), \hat{\Psi}(x, t, \theta)=z \geq 0>\hat{z}, z$ finite $\}$. Compute the point $x^{T}=\sup G(x(t, \theta)) \in[0, g(K, \theta)]$, which exists in $[0, g(K, \theta]$ as $\left[0, g(K, \theta]\right.$ is a complete sublattice of $R_{+}^{J}$. Compute $z^{T}=\hat{\Psi}\left(x^{T}, t^{\prime}, \theta\right) \geq z \geq 0$ where the inequality $z^{T} \geq z$ follows from the definition of join $\vee G(x(t, \theta))$ and the fact that $\hat{\Psi}$ is strictly increasing in $x$. By the isotonicity and continuity of $\hat{\Psi}$ in $x$, define $\hat{G}(x(t, \theta))=\left\{x \mid z^{T} \geq \hat{\Psi}(x, t, \theta) \geq 0>\hat{z}\right\}$ where, by construction, $x^{T}=\sup \hat{G} \subset[0, g]$. Also, note that, $\hat{G}(x(t, \bar{\theta}))$ is nonempty (as certainly we have $x(t, \theta) \in G(x(t, \theta))$, and therefore, so is $\hat{G}(x(t, \theta))$.

Claim B: The correspondence $x_{Z}^{*}(t, \theta): \mathbf{T} \times \boldsymbol{\Theta} \rightarrow \mathbf{2}^{\mathbf{K}^{J}} \backslash \varnothing$ is ascending in Smithson's weak set relation (C1) and (C2).

Proof: Smithson's weak induced set relations (C1) and (C2) are discussed in Section 4. For $t \in \mathbf{T}_{+}$, by Lemma 7 , as $\hat{\Psi}$ is (i) strictly increasing and continuous in $x$ on $[0, g)$ and nondecreasing and upper semicontinuous in $x$ on all of $\mathbf{R}_{+}^{J}$, (ii) the order interval $[0, g(K, \theta)]$ is convex (and, therefore, connected), and (iii) by lemma $7(\mathrm{i})$-(ii), $\hat{\Psi}\left(x^{*}(t, \theta), t^{\prime}, \theta\right) \leq 0$ for $t^{\prime} \geq t$, following the argument in Claim A by letting the lower solution be $x_{L}\left(t^{\prime}, \theta\right)=x^{*}(t, \theta)$, and by lemma 7 (iii) noting there exists an upper solution $x^{T}\left(t^{\prime}, \theta\right)$ such that $\hat{\Psi}\left(x^{T}, t^{\prime}, \theta\right) \geq 0$, we can again apply the theorem of Guillerme's ([34], Theorem 3$)$ to $\hat{\Psi}(x, t, \theta)$ relative to the connected suborder interval $\left[x_{L}\left(t^{\prime}, \theta\right), x^{T}\left(t^{\prime}, \theta\right)\right]=\left[x(t, \theta), x^{T}\left(t^{\prime}, \theta\right)\right] \subset$ $\left(0, g(K, \theta) \subset R_{+}^{J}\right.$. We conclude for any $x(t, \theta) \in x^{*}(t, \theta)$, there exists a element $x\left(t^{\prime}, \theta\right) \in x^{*}\left(t^{\prime}, \theta\right)$ such that $x(t, \theta) \leq x\left(t^{\prime}, \theta\right)$. This proves $x^{*}(t, \theta)$ is ascending in Smithson's weak set relation (C1) on $\mathbf{T}_{+}$. A similar argument shows that for any $x\left(t^{\prime}, \theta\right) \in x^{*}\left(t^{\prime}, \theta\right)$, there exists and $x(t, \theta) \in x^{*}(t, \theta)$ that minorizes $x\left(t^{\prime}, \theta\right)$. This proves $x^{*}(t, \theta)$ is ascending to $\mathbf{K}^{J}$ in Smithson's weak set relation (C2) on $\mathbf{T}_{+}$.

\footnotetext{
${ }^{29}$ Let $X$ be a partially ordered set. We say a set $X_{1} \subset X$ is antichained if no two elements of $X_{1}$ are ordered.
} 
Noting the definition of $x_{Z}^{*}(t, \theta)$ in (7) that extends $x_{Z}^{*}(t, \theta)$ onto all of $\mathbf{T} \times \Theta$, we have (i) $x_{Z}^{*}: \mathbf{T}_{+} \times \Theta \rightarrow \mathbf{K}^{J}$ ascending in Smithson's weak set relation (C1), and for any $t^{\prime} \in \mathbf{T} \backslash \mathbf{T}_{1}, t^{\prime} \geq t, x_{Z}^{*}(t, \theta)$ is a singleton with $x_{Z}^{*}(t, \theta) \leq x_{Z}^{*}\left(t^{\prime}, \theta\right)$ for all $t \in \mathbf{T}_{1}$ and fixed $\theta \in \Theta$. We conclude $x_{Z}^{*}(t, \theta): \mathbf{T} \times \Theta \rightarrow \mathbf{K}^{J}$ is a nonempty correspondence that is ascending in the Smithson weak set relation $(\mathrm{C} 1)$ on $\mathbf{T} \times \Theta$

A similar argument shows $x_{Z}^{*}: \mathbf{T} \times \boldsymbol{\Theta} \rightarrow \mathbf{K}^{J}$ is ascending in Smithson's weak set relation $(\mathrm{C} 2)$.

Claim $C: x_{Z}^{*}(t, \theta): \mathbf{T} \times \boldsymbol{\Theta} \rightarrow \mathbf{2}^{\mathbf{K}^{J}}$ is anti-chained valued (and therefore chain subcomplete valued) for each $(t, \theta)$.

Proof: Recall that by remark above, we first note that $x_{Z}^{*}: \mathbf{T}_{1} \times \Theta$ is antichain-valued. It is therefore chain subcomplete-valued on $\mathbf{T}_{1} \times \Theta$. Given the definition of the extension of $x_{Z}^{*}(t, \theta)$ to $\mathbf{T} \times \Theta$ provided in (7), we conclude that $x_{Z}^{*}: \mathbf{T} \times \boldsymbol{\Theta} \rightarrow \mathbf{K}^{J}$ is antichained valued. Therefore $x_{Z}^{*}(t, \theta)$ is chain subcomplete for each $(t, \theta) \in \mathbf{T} \times \mathbf{\Theta}$.

Then by Claims A-C, $x_{Z}^{*}(t, \theta)$ satisfies the hypotheses of Lemma 8 , and therefore $x_{Z}^{*}(t, \theta)$ admits an isotone selection. Therefore $A h$ in (7) and (8) is well-defined.

(ii) We note that by part (i) of the proposition, $A h(K, \theta)$ isotone in $K$ with $A h(0, \theta)=0$ for all $\theta$ by definition in (8). We therefore have when $K^{\prime} \geq K$ and $K^{\prime} \neq 0$

$$
-\hat{\Psi}_{1}\left(A h\left(K^{\prime}, K^{\prime}, \theta, h\right)\right)+\Psi_{2}(A h(K, \theta)) \geq-\hat{\Psi}_{1}(A h(K, K, \theta, h))+\Psi_{2}(A h)=0
$$

As each $\Psi_{j 2}(x)$ is only a function of $g_{j}-A h_{i}(K, \theta)$ for each $j$, by the concavity of the vector of marginal utilities $u(c)$ we have $A h(K, \theta)$ such that for each $j, g_{j}-A h_{j}$ is isotone in $K$, each $\theta$. As $\Theta$ is finite, and both $A h$ and $g-A h$ are isotone in $K$, and $g$ is uniformly continuous in $K, A h$ is an element of an equicontinuous set of functions on the compactum $\mathbf{S}$ ). Therefore $A h \subset \mathbf{H}$.

(iii) Continuity follows from an standard argument for operators on compact subsets of $\mathbf{C}^{+}(\mathbf{S})$ where $\mathbf{S}$ is compact (e.g, Coleman [19], Proposition 4). Further, as $A\left(H_{1}\right)=\left\{A h \mid h \in H_{1} \subset \mathbf{H}\right\} \subset \mathbf{H}$ is relative compact for all $H_{1} \subset \mathbf{H}$, the closure of $A\left(H_{1}\right)$ is compact. Therefore $A h$ is continuous and compact in $h$, i.e., completely continuous in $h$.

(iv) Consider any $h^{\prime} \geq h$, with $h, h^{\prime} \in \mathbf{H}$. Since $\Psi$ is increasing in $h$ and $\Psi(A h, K, \theta, h)=0$ by definition of the operator $A$, then $Z(A h, K, \theta, h) \geq 0$. Consequently, with $\Psi$ is strictly increasing in its first argument, by an argument similar to (i) for the existence of monotone selection, it must be that $A h^{\prime}(K, \theta) \geq$ $A h(K, \theta)$ for all $(K, \theta)$.

(v) Consider an increasing net $H \subset \mathbf{H}, h^{u}=\sup (H)$. The point $h^{u}$ is welldefined as $\mathbf{H}$ is a complete lattice. As $A h$ is topologically continuous (in the uniform metric topology), for any increasing sequence $h_{i \in I}$ in $H$ such that $h_{i} \rightarrow$ $h=h^{u}, A\left(h_{i \in I}\right) \rightarrow A\left(h^{u}\right)$. Further, as $\mathbf{H}$ is equicontinuous, we have $\vee h_{i \in I}=$ $h=h^{u}$ (see for example, Heikkila and Reffett [37], Lemma 4.1). Since $A h$ 
isotone, and $A\left(h_{i \in I}\right)$ a chain, we therefore have $\vee A\left(h_{i}\right)=A\left(h^{u}\right)=A\left(\lim h_{i \in I}\right)$. As $h^{u}=\vee\left(h_{i}\right)=\vee H$, we have $A(\vee H)=\vee\left(A\left(h_{i \in I}\right)\right)=\vee A(H)$. As similar argument works for a decreasing chain $h_{i \in I} \rightarrow h^{u}$ for $A\left(\wedge h_{i}\right)=\wedge A\left(h_{i \in I}\right)=$ $\wedge A\left(h^{u}\right)$. Therefore $A h$ is order continuous along sequences in $\mathbf{H}$.

We now use Proposition 2 to prove Theorem 3:

Proof of Theorem 3:

(i) The set $\mathbf{H}$ is a nonempty complete lattice by the first lemma in Section Four, and the operator $A$ is a isotone self map on $\mathbf{H}$. Therefore by the main theorem in Tarski ( [77], Theorem 1), $E(\pi)$ is nonempty complete lattice for each $\pi$.

(ii) First we note that $A h$ by Proposition 2(ii), $A\left(H_{1}\right)=\left\{A h \mid h \in H_{1} \subset\right.$ $[0, g]\}$ is relatively compact as $A h$ is a compact operator (e.g., $A$ maps bounded subsets of $[0, g]$ into relatively compact sets of the order interval $[0, g]$ ); by Proposition 2(iii) $A h$ is completely continuous; and by Proposition 2(iv) $A h$ is order continuous. That $A(g)=g$ is by definition the maximal fixed point. By the lemma in Vulikh [81] cited above, the successive approximations $\lim _{n} A^{n} 0$ converges to a minimal fixed point $h_{l}^{*}=\inf E(\pi) \geq 0$. The convergence is uniform as the sequence is equicontinuous and the operator is completely continuous (see Amann ([2], Corollary 6.2).

(iii) The minimal fixed point $h_{l}^{*} \in E(\pi)$ is such that $g-h_{l}^{*}>0$ follows from an obvious modification of the main theorem in Greenwood and Huffman [33], where $A^{n} 0$ is the optimal plan for the economic agents along equilibrium trajectories $n$ periods from her terminal date (along an equilibrium trajectory where $k=K), T^{n} J_{i 0}$ is the value function associated with the Bellman operator for agent $i$, with $J_{i 0}=0$, and the Inada condition in Assumption Three implying that $\lim _{n} A^{n}(0)<g$.

(iv). First we note by Tarski's theorem, $E(\pi)$ is complete lattice valued for each $\pi$. Let $\pi^{\prime} \leq \pi$ with $\pi_{k}^{\prime} \leq \pi_{k}, \pi_{n}^{\prime}=\pi_{n}$ (implying the return on capital $\bar{r}$ falls under $\pi_{k}^{\prime}$ pointwise). Notice we have $A g=g$ for all $\pi \in \Pi$. From the definition of $Z$ in (7) (and its implied implications on the system $\hat{\Psi}$ in Proposition 2), when $\pi_{k}^{\prime} \leq \pi_{k}$, we have $Z\left(\pi_{k}^{\prime} ; h, A h\left(\pi_{k} ; K, \theta\right), K, \theta\right) \geq Z\left(\pi_{k} ; h, A h\left(\pi_{k} ; K, \theta\right), K, \theta\right)=0$ where now we make the dependence of $A h$ on $\pi_{k}$ explicit by adding it as an argument to $Z$ in (7). Then by Lemma 7 (iii), $A h\left(\pi_{k}^{\prime} ; K, \theta\right) \leq A h\left(\pi_{k} ; K, \theta\right)$. By an argument Topkis ([78], Theorem 2.5.2) (and noting the dual order on $\Pi$ ), we have (i) $E(\pi)$ is isotone in the strong set order, i.e., $E\left(\pi_{k}^{\prime}\right) \geq_{s} E\left(\pi_{k}\right)$ when $\pi^{\prime}=\left(\pi_{k}^{\prime}, \pi_{n}\right) \geq\left(\pi_{k}, \pi_{n}\right)=\pi$ with the dual order on $\Pi$; and (ii) $\sup E(\pi)$ and $\inf E(\pi)$ forming isotone selections

The proof of Theorem 5 concerning the fixed points of $A^{c} h$ in (15) and (16) of Section 6 requires a version of Proposition 2 adapted for the expanded parameter space used to define $Z_{c}$ in equations (12) and (14). We remark the arguments here are basically exactly as in the proof of Lemma 7, Proposition 
2, and Theorem 3. We first state this version of Proposition 2 as Proposition 9 (which is used in the proof of Theorem 5 just as Proposition 2 is used in the proof of Theorem 3).

Proposition 9 Under Assumptions One, Two, and Four, (i) for any $h \in \mathbf{H}$ and $(K, \hat{K}, \theta) \in \mathbf{K}^{2 J} \times \Theta, A h(K, \hat{K}, \theta)$ is well-defined; (ii) $A h(K, \hat{K}, \theta) \subset \mathbf{H} ;($ iii $)$

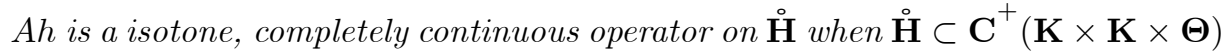
(e.g., space of continuous functions on $\mathbf{K} \times \mathbf{K} \times \boldsymbol{\Theta}$ equipped with the uniform topology and pointwise Euclidean order); (iv) Ah is order-continuous on $\hat{\mathbf{H}}$.

Proof: Following the proof of Proposition 2, first rewrite the system of Euler inequalities in (12) and (14) by defining a new mapping $\hat{Z}_{c}(x, K, \hat{K}, h, \theta)$ as in the proof of Proposition 2 above (e.g., $\hat{Z}_{c}$ will be the mapping $Z_{c}$ defined in (12) and (14) with the addition of a new set of KKT multipliers defined in equilibrium by a new mapping $\phi_{c}(x, K, \hat{K}, h, \theta)$ exactly as in the proof of Proposition 2.

For the details, let $t_{c}=(K, \hat{K}, h) \in \stackrel{\circ}{\mathbf{T}}_{+}=\mathbf{K}_{+}^{J} \times \mathbf{K}_{+}^{J} \times \stackrel{\circ}{\mathbf{H}} \backslash \mathbf{g}$, and define extended real valued system $\hat{\Psi}_{c}(x, K, \hat{K}, h, \theta): \mathbf{K}^{J} \times \stackrel{\circ}{\mathbf{T}}_{+} \times \Theta \rightarrow \overline{\mathbf{R}}^{J}$ as $\rightarrow \overline{\mathbf{R}}^{J}$ :

$$
\begin{aligned}
\hat{\Psi}_{c}\left(x, t_{c}, \theta\right) & =\Psi_{2}(x, K, \theta)-\hat{\Psi}_{1}\left(x, t_{c}, \theta\right) \text { and } \\
\hat{\Psi}_{1 c} & \left.=\beta \int_{\Theta} u^{\prime}\left(g\left(x, \theta^{\prime}\right)-h\left(x, \theta^{\prime}\right)\right)\right) \bar{r}\left(x, \theta^{\prime}\right) \chi\left(\theta, d \theta^{\prime}\right)+\phi_{c}\left(x, t_{c}, \theta\right) I_{x}(x=0), \\
\Psi_{2 c} & =u^{\prime}(g(K, \theta)-x),
\end{aligned}
$$

where again we use the shorthand $I_{x}(x=0)$ is the indicator variable on vector of equations whose components indicate in each equation $j \in \mathbf{J}$ whether household $j$ is borrowing constrained, i.e., $I_{x_{j}}=1$ when $x_{j}=0$. Here $\phi_{c}\left(x, t_{c}, \theta\right)$ : $\mathbf{K}^{J} \times \mathbf{T}_{+} \times \boldsymbol{\Theta} \rightarrow \overline{\mathbf{R}}^{J}$ defined componentwise for each $j \in J$ as follows:

$$
\phi_{j c}\left(x, t_{c}, \theta\right)=\Psi_{j 2 c}(x, K, \theta)-\Psi_{j 1 c}\left(x, t_{c}, \theta\right) \text { for } j=1,2, \ldots, J .
$$

where $\Psi_{1 c}\left(x, t_{c}, \theta\right)$ and $\Psi_{2 c}\left(x, t_{c}, \theta\right)$ are given by expressions (12) and (14) of Section 6 .

We now follow the exact same construction as Proposition 2: that is,

Claim A: The correspondence $x_{Z c}^{*}\left(t_{c}, \theta\right): \stackrel{\circ}{\mathbf{T}} \times \Theta \rightarrow \mathbf{2}^{\mathbf{K}^{J}}$ is nonempty for each $\left(t_{c}, \theta\right) \in \stackrel{\mathrm{T}}{\times} \Theta$.

Proof: To prove $x_{Z c}^{*}\left(t_{c}, \theta\right)$ is nonempty on $\stackrel{\mathrm{T}}{+}_{+} \times \Theta$, we note that we have $\hat{\Psi}_{c}$ strictly increasing and continuous in $x$ on the interior of $[0, g(K, \hat{K}, \theta))$, therefore for each $\left(t_{c}, \theta\right) \in \stackrel{\circ}{\mathbf{T}}_{+} \times \Theta$ there is a pair of vectors $\left(x_{l}\left(t_{c}, \theta\right), x^{T}\left(t_{c}, \theta\right)\right) \in$ $[0, g(K, \hat{K}, \theta)] \times[0, g(K, \hat{K}, \theta)]$ such that $(\mathrm{a}) \hat{\Psi}\left(x_{l}\left(t_{c}, \theta\right), t_{c}, \theta\right) \leq 0 ;(\mathrm{b}) \hat{\Psi}\left(x^{T}\left(t_{c}, \theta\right), t_{c}, \theta\right) \geq$ 0 , and (c) $x_{l}\left(t_{c}, \theta\right) \leq x^{T}\left(t_{c}, \theta\right)$ in the standard component product Euclidean order on $R^{J}$, and (iii) $x_{l}\left(t_{c}, \theta\right) \neq 0$.As $\hat{\Psi}$ is strictly increasing and continuous jointly in $x$ on $(0, g(K, \hat{K}, \theta))$ and nondecreasing and upper-semicontinuous 
on all of (connected) order interval $[0, g(K, \hat{K}, \theta)]$, it is is nondecreasing in $x_{j}$ on $\left[0, g_{j}(K, \hat{K}, \theta)\right]$ for fixed $x^{j}=\left(x_{1}, x_{2}, \ldots x_{j-1}, x_{j+1}, \ldots, x_{J}\right), x=\left(x_{j}, x^{j}\right)$ each $j \in J$. The mapping $\hat{\Psi}\left(x, t_{c}, \theta\right)$ therefore satisfies the semi-continuity conditions in the generalized intermediate value theorem on arbitrary product spaces of Guillerme ([34], Theorem 3) on $\left[x_{L}\left(t_{c}, \theta\right), x^{T}\left(t_{c}, \theta\right)\right]$. Therefore we conclude that correspondence $x_{Z c}^{*}\left(t_{c}, \theta\right)$ is nonempty on the connected suborder interval $\left[x_{l}\left(t_{c}, \theta\right), x^{T}\left(t_{c}, \theta\right)\right] \subset[0, g(K, \hat{K}, \theta)) \subset R_{+}^{J}$ with $x_{l}\left(t_{c}, \theta\right) \neq 0 .{ }^{30}$. Therefore we conclude that $x_{Z c}^{*}\left(t_{c}, \theta\right)$ is nonempty for each $\left(t_{c}, \theta\right) \in \mathbf{\mathbf { T }}_{+} \times \Theta$. Notice that by the definition of $x_{Z c}^{*}\left(t_{c}, \theta\right)$ in (15) and (16) is well defined for any $\left(t_{c}, \theta\right) \in \stackrel{\circ}{\mathbf{T}} \backslash \stackrel{\circ}{\mathbf{T}}_{+} \times \Theta$. Therefore $x_{Z c}^{*}\left(t_{c}, \theta\right): \stackrel{\circ}{\mathbf{T}} \times \boldsymbol{\Theta} \rightarrow \mathbf{K}^{J}$ is well-defined. $\mathbf{v}$

Remark: Before we proceed to Claim B, we note that all the additional remarks concerning some additional properties of the correspondence $x_{Z}^{*}(t, \theta)$ also hold for $x_{Z c}^{*}\left(t_{c}, \theta\right)$. That is, as in Proposition 2, the correspondence $x_{Z c}^{*}$ is antichain-valued for each $\left(t_{c}, \theta\right) \in \stackrel{\mathbf{T}}{+}_{+} \times \Theta$ relative to the standard componentwise order on $R_{+}^{J}$; and the uppersets $\left.G\left(x\left(t_{c}, \theta\right)\right)\right)=\left\{x \mid x \geq x\left(t_{c}, \theta\right), \hat{\Psi}_{c}\left(x, t_{c}, \theta\right)=\right.$ $z \geq 0>\hat{z}, z$ finite $\}$ and $\hat{G}\left(x\left(t_{c}, \theta\right)\right)=\left\{x \mid z^{T} \geq \hat{\Psi}_{c}\left(x, t_{c}, \theta\right) \geq 0>\hat{z}\right\}$ are again well defined (e.g, nonempty).

Claim B: The correspondence $x_{Z c}^{*}\left(t_{c}, \theta\right): \stackrel{\circ}{\mathbf{T}} \times \mathbf{\Theta} \rightarrow \mathbf{2}^{\mathbf{K}^{J}} \backslash \varnothing$ is ascending in $(K, h)$ for each $(\hat{K}, \theta)$, and ascending in $\hat{K}$ for each $(K, h, \theta)$ in Smithson's weak set relation (C1) and (C2) when $\hat{K} \in \mathbf{K}^{J}$, given the dual Euclidean partial order.

Proof: The argument follows from proof of claim B follows directing from Proposition 2, Claim B if you order $\hat{K} \in \mathbf{K}^{J}$ with the dual order, using $t=t_{c} \cdot \boldsymbol{\nabla}$

Claim $C: x_{Z c}^{*}\left(t_{c}, \theta\right): \stackrel{\circ}{\mathbf{T}} \times \mathbf{\Theta} \rightarrow \mathbf{2}^{\mathbf{K}^{J}}$ is anti-chained valued (and therefore chain subcomplete valued) in $(t, \theta)$.

Proof: Follows from $\hat{\Psi}_{c}$ is strictly increasing in $x$ on $\mathbf{K}^{J}$ using $t=t_{c} \cdot \mathbf{\nabla}$

Then by Claims A-C, $x_{Z c}^{*}\left(t_{c}, \theta\right)$ satisfies the hypotheses of lemma 8 , and therefore $x_{Z c}^{*}\left(t_{c}, \theta\right)$ admits an isotone selection in $(K, h)$ and an antitone selection in $\hat{K}$ for each $\theta \in \Theta$. Therefore $A^{c} h$ in (15) and (16) is well-defined.

(ii) We note that by part (i) of the proposition, $A h(K, \hat{K}, \theta)$ isotone in $(K, \hat{K})$ when $\hat{K} \in \mathbf{K}^{J}$ is given the dual partial order. We also have $A h(0, \theta)=0$ for all $\theta$ by definition in (15) and (16). We therefore have when $K^{\prime} \geq K$

$-\hat{\Psi}_{1}\left(A h\left(K^{\prime}, \hat{K}^{\prime}, \theta\right)+\Psi_{2}(A h(K, \hat{K}, \theta)) \geq-\hat{\Psi}_{1}(A h(K, \hat{K}, \theta))+\Psi_{2}(A h(K, K, \theta)=0\right.$

As each $\Psi_{j 2}(x)$ is only a function of $g_{j}(K, \hat{K}, \theta)-A h_{i}(K, \hat{K}, \theta)$ for each $j$, by the concavity of the vector of marginal utilities $u(c)$ we have $A h(K, \hat{K}, \theta)$ such that for each $j, g_{j}-A h_{j}$ is isotone in $K$, antitone in $\hat{K}$ for each $\theta$. As $\Theta$ is finite, and both $A h$ and $g-A h$ are isotone in $K$, and $g$ is uniformly continuous in $K$,

${ }^{30}$ Using Guillerme's notation, take $\hat{\Psi}_{1}=g, \Psi_{2}=f$, to apply the theorem. 
$A h$ is an element of an equicontinuous set of functions on the compactum $\mathbf{S}$ ). Therefore $A h \subset \mathbf{H}$.

(iii)-(v). See proof of Proposition 2 for a similar argument noting the expanded state-space.

\section{References}

[1] Aiyagari, R. 1994. Uninsured idiosyncratic risk and aggregate saving. Quarterly Journal of Economics, 109, 659-684.

[2] Amann, H. 1976. Fixed point equations and nonlinear eigenvalue problems in order Banach spaces. SIAM Review,18, 4, 620-709.

[3] Amann, H. 1977. Order Structures and Fixed Points.SAFA 2, ATTI del $2 \circ$ Seminario di Analisi Funzionale e Applicazioni. MS..

[4] Becker, R. 1980. On the long-run steady state in a simple dynamic model of equilibrium with heterogeneous households. Quarterly Journal of Economics, 95, 375-382

[5] Becker, R. 1985. Comparative dynamics in aggregate models of optimal capital accumulation. Quarterly Journal of Economics, 100, 1235-1256.

[6] Becker, R. 1985. Capital income taxation and perfect foresight. Journal of Public Economics, 26, 147-167.

[7] Becker, R. and J. Boyd. 1997. Capital Theory, Equilibrium Analysis, and Recursive Utility. Basil Blackwell, Cambridge.

[8] Becker R, and I. Zilcha. 1997. Stationary Ramsey equilibria under uncertainty. Journal of Economic Theory, 75, 122-140.

[9] Becker, R. and C. Foias. 1998. Implicit programming and the invariant manifold for Ramsey equilibria. in Yuri Abramovich, Evgenios Avgerinos, and Nicolas Yannelis, eds. Functional Analysis and Economic Theory, 1998, Springer-Verlag.

[10] Becker, R. and B. Chen. 2001. Implicit programming and computing the invariant manifold for dynamic Ramsey equilibrium Problems. MS. Indiana University.

[11] Berge, C. 1963. Topological Spaces, MacMillan Press.

[12] Bergin, J and D Bernhardt. 1992. Anonymous sequential games with aggregate uncertainty. Journal of Mathematical Economics, 21, 543-562. 
[13] Bewley, T. 1986. Stationary monetary equilibrium with a continuum of independently fluctuating consumers. in Contributions to Mathematics in Honor of Gerard Debreu, ed. W. Hildenbrand and A. Mas-Colell. NorthHolland, Amsterdam.

[14] Bhattacharya, R. and O. Lee. 1988. Asymptotics of certain Markov processes which are not in general irreducible. Annals of Probability, 16, 1333-1347

[15] Bizer, D. and K. Judd. 1989. Taxation and uncertainty. American Economic Review, 78, 331-336.

[16] Birkhoff, G. 1967. Lattice Theory. AMS Press.

[17] Brock, W. and L. J. Mirman. 1972. Optimal growth and uncertainty: the discounted case. Journal of Economic Theory, 4, 479-513.

[18] Coleman, W. J., II. 1990. Solving the stochastic growth model by policy function iteration, Journal of Business and Economic Statistics, 8, 27-29.

[19] Coleman, W. J., II. 1991. Equilibrium in a production economy with an income tax. Econometrica, 59, 1091-1104.

[20] Coleman, W. J., II. 1997. Equilibria in distorted infinite-horizon economies with capital and labor. Journal of Economic Theory, 72, 446-461.

[21] Coleman, W. J., II. 2000. The uniqueness of an equilibrium in distorted infinite horizon economies with capital. Journal of Economic Theory, 95, 71-78.

[22] Dacic, R. 1979. Fixed points of antitone mappings in conditionally complete partially ordered sets. Publications De L'Institut Mathematique, 40, 83-90.

[23] Datta, M. and K. L. Reffett. 2005. Isotone recursive methods. forthcoming in The Handbook of Optimal Growth, vol. 1: The discrete time horizon model, eds. C. LeVan, T. Mitra, and K. Nishimura, Kluwer Press.

[24] Datta, M., L. J. Mirman and K. L. Reffett. 2002, Existence and uniqueness of equilibrium in distorted dynamic economies with capital and labor, Journal of Economic Theory, 103, 377-410.

[25] Datta, M. L. J. Mirman, O. F. Morand and K. L. Reffett. 2002. Monotone methods for Markovian equilibrium in dynamic economies, Annals of $\mathrm{Op}$ erations Research, 114, 117-144.

[26] Danthine, J.-P., and J. Donaldson 1981. Stochastic properties of fast vs slow growing economies, Econometrica, 49, 1007-1033.

[27] Davey, B. A., and H. A. Priestley. 1990. Introduction to Lattices and Order. Cambridge Press. 
[28] Dubins, L. and D. Freedman. 1966. Invariant distributions for certain Markov processes. Annals of Mathematical Statistics, 37, 837-848.

[29] Duffie, D., J. Geanakoplos, A. MasColell and A. McLellan. 1994. Stationary Markov equilibria. Econometrica, 62, 745-781.

[30] Dugundji, J and V. Granas. 1982. Fixed Point Theory, Polish Scientific Press.

[31] Duran, J. 2000. On dynamic programming with unbounded returns. Economic Theory, 15, 339-352.

[32] Frink, Orrin, Jr. 1942. Topology in lattices. Transactions of the American Mathematical Society, 51, 569-582.

[33] Greenwood, J., and G. Huffman. 1995. On the existence of nonoptimal equilibria in dynamic stochastic economies, Journal of Economic Theory, 65, 611-623.

[34] Guillerme, J. 1995. Intermediate value theorems and fixed point theorems for semi-continuous functions in product spaces. Proceedings of the American Mathematical Society, 123, 2119-2122.

[35] Guo, D. and V. Lakshmikantham. 1988. Nonlinear Problems in Abstract Cones. Academic Press.

[36] Heikkila, S. and V. Lakshmikantham. 1994. Monotone iterative techniques for discontinuous nonlinear differential equations, Marcel Dekker.

[37] Heikkila, S. and K. Reffett. 2004. Fixed point theorems for multivalued functions. MS. Arizona State University.

[38] Heikkila, S. and K. Reffett. 2004. Multivalued generalizations of classical fixed point theorems. MS. Arizona State University.

[39] Hopenhayn, H., and E. Prescott. 1992. Stochastic monotonicity and stationary distributions for dynamic economies. Econometrica, 60, 1387-1406.

[40] Huggett, M. 1997. The one-sector growth model with idiosyncratic shocks. Journal of Monetary Economics, 39, 385-403.

[41] Hutson, V, and J. Pym. 1980. Applications of Functional Analysis and Operator Theory, Academic Press.

[42] Judd, K. 1992. Projection methods for solving aggregate growth models. Journal of Economic Theory, 58, 410-452.

[43] Judd, K. and J. Gasper. 1997. Solving large rational expectations models. Macrodynamics, 1, 45-75. 
[44] Judd, K. F. Kubler, and K. Schmedders. 2000. Computing equilibria in infinite horizon finance economies: the case of one-asset", Journal of Economic Dynamics and Control, 24, 1047-1078.

[45] Judd, K. F. Kubler, and K. Schmedders. 2003. Asset trading in infinite horizon economies with dynamically complete markets and heterogeneous agents. Journal of Finance. 2203-2217.

[46] Kehoe, T. 1991. Computation and multiplicity of equilibrium. in W. Hildenbrand and H. Sonnenschein, eds. Handbook of Mathematical Economics, 2049-2143.

[47] Kehoe, T., P. Romer, and M. Woodford. 1990. Determinacy of equilibria in dynamic economies with finitely many agents, Journal of Economic Theory, $50,1-15$.

[48] Krasnoselskii, M.A., G.M. Vainikko, P.P. Zabreiko, Ya.B. Rutitskii, and V. Ya. Stetsenko. 1972. Approximation Solution of Operator Equations. Wolters-Noordhoff. The Netherlands.

[49] Krebs, T. 2003. Human capital risk and economic growth. Quarterly Journal of Economics, 118, 709-745.

[50] Krebs, T. 2004. Recursive equilibrium in endogenous growth models with incomplete markets. MS. Brown.

[51] Krebs, T. 2004. Non existence of recursive equilibria on compact state spaces when markets are incomplete, Journal of Economic Theory, 115, 134-150.

[52] Krusell, P. and A. Smith. 1998. Income and wealth heterogeneity in the macroeconomy, Journal of Political Economy, 106, 867-896.

[53] Kubler, F. and K. Schmedders. 2003. Stationary equilibrium in asset pricing models with incomplete markets and collateral. Econometrica, 1767-1795.

[54] Kurpel, N.S. 1976. Projection-Iterative Methods for Solution of Operator Equations. Translations of Mathematical Monographs, AMS Press.

[55] Miao, J. 2002. Competitive equilibria in economies with a continuum of consumers and aggregate shocks. MS. Rochester.

[56] Milgrom, P. and C. Shannon. 1994. Monotone comparative statics. Econometrica, 62, 157-180.

[57] Mirman, L. J. and I. Zilcha. 1975. On optimal growth under uncertainty. Journal of Economic Theory ,11, 329-339.

[58] Mirman, L. J., O. F. Morand and K. L. Reffett. 2004. A qualitative approach to Markovian equilibrium in infinite horizon economies with capital. MS. Arizona State University. 
[59] Mirman, L. J., K. L. Reffett and J. Stachurski. 2004. Computing Bewley Models. MS Arizona State University.

[60] Montrucchio, L. 1987. Lipschitz continuous policy functions for strongly concave optimization problems. Journal of Mathematical Economics, 16, $259-273$

[61] Moore, J.C., 1999. Mathematical Methods for Economic Theory 1, Springer.

[62] Morand, O. F. and K. L. Reffett. 2003. Existence and uniqueness of equilibrium in nonoptimal unbounded infinite horizon economies, Journal of Monetary Economics, 50, 1351-1373.

[63] Reffett, K. L. 2004. Computing Markovian equilibrium using Interval Condensing Methods. MS. Arizona State University.

[64] Reffett, K. L. 2004. Mixed Monotone Recursive Methods, MS. Arizona State University.

[65] Rockafellar, R. T. and R. Wets. 1997. Variational Analysis. Springer Press.

[66] Robinson, S. 1982. Generalized equations and their solutions: Part II. Applications to nonlinear programming. Mathematical Programming Study, $19-200-221$.

[67] Romer, P. 1986. Increasing returns and long-run growth. Journal of Political Economy, 94, 1002-1037

[68] Santos, M. 1992. Differentiability and comparative analysis in discrete-time infinite horizon optimization. Journal of Economic Theory, 57, 222-229.

[69] Santos, M. 2000 The numerical accuracy of numerical solutions using Euler residuals. Econometrica, 68, 1377-1402.

[70] Santos, M. 2002. On non existence of Markov equilibria in competitivemarket economies." Journal of Economic Theory, 105, 73-98.

[71] Santos, M. and A. Peralta-Alva. 2004. Accuracy of simulations for stochastic dynamic models. MS. Arizona State University.

[72] Santos, M. and J. Vigo-Aguiar. 1998. Analysis of a numerical dynamic programming algorithm applied to economic models. Econometrica, 66, $409-426$.

[73] Schectman, J. and V.L.S. Escudero. 1977. Some results on an income fluctuation problem. Journal of Economic Theory, 16, 151-166.

[74] Scheinkman, J. and L. Weiss. 1986. Borrowing constraints and aggregate economic activity, Econometrica, 54, 23-46.

[75] Smithson, R.E. 1971. Fixed points of order preserving multifunctions. Proceedings of the American Mathematical Society, 28, 304-310. 
[76] Tarski, A. A fixed point for lattices and its applications. Bull. of Amer. Math. Soc. 55, 1051-52.

[77] Tarski, A. 1955. A lattice-theoretical fixpoint theorem and its applications. Pacific Journal of Mathematics, 5, 285-309.

[78] Topkis, D. M. 1998. Supermodularity and Complementarity, Princeton University Press.

[79] Townsend, R. 1980. Models of Money with Spatially Separated Agents. in J. Karekin and N. Wallace, ed., Models of Monetary Economies, Federal Reserve Bank of Minneapolis.

[80] Veinott, A. 1992. Lattice programming: Qualitative optimization and equilibria. MS. Stanford.

[81] Vulikh, B. 1967. Introduction to the Theory of Partially Ordered Spaces, Noordhoff Scientific Publishers.

[82] Zeidler, E. 1986. Nonlinear Functional Analysis and its Applications, vol. 1, Springer Verlag. 